Supporting Information for

\title{
Theoretical Calculation of Electronic Circular Dichroism of the Rotationally Restricted 3,8"-Biflavonoid Morelloflavone
}

\author{
Yuanqing Ding, ${ }^{\dagger}$ Xing-Cong Li, ${ }^{*}, \dagger$ and Daneel Ferreira ${ }^{*}, \dagger, \dagger$ \\ National Center for Natural Products Research, Research Institute of Pharmaceutical Sciences and \\ Department of Pharmacognosy, School of Pharmacy, The University of Mississippi, University, \\ Mississippi 38677
}

\section{Table of Contents}

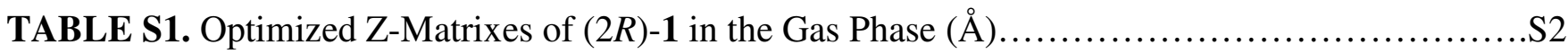

TABLE S2. Optimized Z-Matrixes of Conformers of $(2 R, 3 S)-2$ in the Gas Phase $(\AA) \ldots \ldots \ldots \ldots \ldots \ldots . . . . . . . . .33$

TABEL S3. Frequencies of $(2 R)-\mathbf{1}$ at B3LYP/6-31G* Level in the Gas Phase $\left(\mathrm{cm}^{-1}\right) \ldots \ldots \ldots \ldots \ldots . . . . . . . .212$

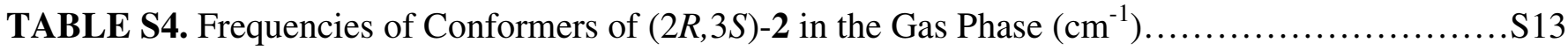

TABLE S5. Dihedral Angles of Conformers of (2R,3S)-2 Optimized at B3LYP/6-31G*

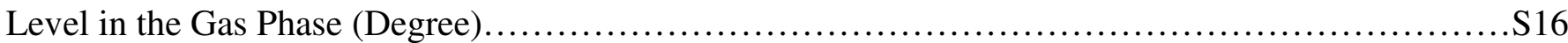

TABLE S6. Important Thermodynamic Parameters of $(2 R, 3 S)-2$ in the Gas Phase and in Methanol Solution (au)

FIGURE S1. Theoretically calculated ECD spectra of $(2 R)-1$ by TDDFT at

BHLYP/6-31G* and BLYP/6-31G* and its experimental ECD (in the region 260-350 nm)....

FIGURE S2. Optimized geometries of conformers of $(2 R, 3 S)-2$ at B3LYP/6-31G* level

in the gas phase

FIGURE S3. Theoretically calculated ECD spectra of $(2 R, 3 S)-\mathbf{2}$ and their weighted ECD

spectra in the gas phase at B3LYP/6-31G* level and those of conformer $\mathbf{2 b 7}$ in the gas

phase at B3LYPP/6-31G* level and in methanol with COSMO model.

S20 
TABLE S1. Optimized Z-Matrixes of $(2 R)-\mathbf{1}$ in the Gas Phase ( $(\AA)$

\begin{tabular}{|c|c|c|c|c|c|c|c|}
\hline \multicolumn{4}{|c|}{$(2 R)-1$ at B3LYP/6-31G* level } & \multicolumn{4}{|c|}{$(2 R)-1$ at B3LYP/6-311++G** level } \\
\hline $\mathrm{O}$ & -0.080076 & 0.061175 & -0.867804 & $\mathrm{O}$ & 0.284653 & -0.823694 & -0.001908 \\
\hline $\mathrm{C}$ & 1.086627 & -0.478241 & -0.206565 & $\mathrm{C}$ & 1.090099 & 0.306036 & 0.413142 \\
\hline $\mathrm{C}$ & 1.268535 & 0.186741 & 1.162491 & $\mathrm{C}$ & 0.663068 & 1.563335 & -0.347622 \\
\hline $\mathrm{C}$ & 0.009268 & 0.059054 & 2.001347 & $\mathrm{C}$ & -0.825232 & 1462 & -0.206189 \\
\hline $\mathrm{C}$ & -1.235831 & 0.018881 & 1.266186 & $\mathrm{C}$ & -1.649411 & 0.636595 & -0.027516 \\
\hline $\mathrm{C}$ & -2.493419 & 0.027130 & 1.946190 & $\mathrm{C}$ & -3.073168 & 0.732922 & 0.002237 \\
\hline $\mathrm{C}$ & -3.686164 & 0.093961 & 1.229923 & $\mathrm{C}$ & -3.859764 & -0.410625 & 0.070547 \\
\hline $\mathrm{C}$ & -3.637593 & 0.157685 & -0.164306 & $\mathrm{C}$ & 8502 & -1.657214 & 6095 \\
\hline $\mathrm{C}$ & -2.422226 & 0.141163 & -0.868157 & $\mathrm{C}$ & -1.844012 & -1.794292 & 0.089064 \\
\hline $\mathrm{C}$ & -1.236731 & 0.068569 & -0.150521 & $\mathrm{C}$ & -1.063035 & -0.651104 & 0.025671 \\
\hline $\mathrm{C}$ & 2.274980 & -0.289359 & -1.121123 & $\mathrm{C}$ & 2.542751 & -0.050853 & 0.210012 \\
\hline $\mathrm{C}$ & 3.260245 & -1.279911 & -1.193161 & $\mathrm{C}$ & 3.487265 & 0.311036 & 1.173006 \\
\hline $\mathrm{C}$ & 4.35 & -1.095761 & -1.9 & $\mathrm{C}$ & 648 & 386 & 661 \\
\hline $\mathrm{C}$ & 4.548277 & 0.079395 & -2.721719 & $\mathrm{C}$ & 5.259572 & -0.604543 & 6205 \\
\hline $\mathrm{C}$ & 653 & 129 & -2.66 & $\mathrm{C}$ & 789 & 78227 & 769 \\
\hline $\mathrm{C}$ & 2.434768 & 0.887849 & -1.862532 & $\mathrm{C}$ & 2.969281 & -0.699841 & 2804 \\
\hline $\mathrm{O}$ & 0.071096 & 0.029806 & 3.241 & $\mathrm{O}$ & -1.2 & 2.956810 & 558 \\
\hline $\mathrm{O}$ & -2.543205 & -0.014548 & 3.283380 & $\mathrm{O}$ & -3.674033 & 1.927290 & 1089 \\
\hline $\mathrm{O}$ & -4.82 & 0.222343 & -0.8 & $\mathrm{O}$ & 2667 & -2.743397 & 490 \\
\hline $\mathrm{H}$ & 0.908594 & -1.552831 & -0.05 & $\mathrm{H}$ & 859 & 0.458428 & 445 \\
\hline $\mathrm{H}$ & 1.49 & 327 & 1.0 & $\mathrm{H}$ & 0.8 & 1.4 & 222 \\
\hline $\mathrm{H}$ & 2.110689 & -0.25 & 1.7 & $\mathrm{H}$ & 307 & 7730 & 195 \\
\hline $\mathrm{H}$ & -1.602214 & -0.025029 & 3.606 & $\mathrm{H}$ & -2.965968 & 2.611810 & 539 \\
\hline $\mathrm{H}$ & -4.636883 & 0.104513 & 1.748 & $\mathrm{H}$ & -4.938090 & -0.332310 & 214 \\
\hline $\mathrm{H}$ & -4.667468 & 0.268526 & -1.771314 & $\mathrm{H}$ & -3.526195 & -3.550223 & 825 \\
\hline $\mathrm{H}$ & -2.390443 & 0.163542 & -1.95 & $\mathrm{H}$ & -1.365958 & -2.765428 & 349 \\
\hline $\mathrm{H}$ & 3.138923 & -2.202357 & -0.629224 & $\mathrm{H}$ & 3.165190 & 0.802907 & 534 \\
\hline $\mathrm{H}$ & 5.15 & -1.8 & -2.0 & $\mathrm{H}$ & 75 & 0.327591 & 155 \\
\hline $\mathrm{H}$ & 5.427492 & 0.222207 & -3.344028 & $\mathrm{H}$ & 629 & -0.821373 & 9591 \\
\hline $\mathrm{H}$ & 3.67 & 1.9 & -3.2 & $\mathrm{H}$ & 4.6 & -1. & 350 \\
\hline $\mathrm{H}$ & 1.663930 & 1.651381 & -1.825178 & $\mathrm{H}$ & 2.240583 & -1.002286 & -1.695576 \\
\hline \multicolumn{4}{|c|}{$(2 R)-1$ at $\mathrm{BLYP} / 6-31 \mathrm{G}^{*}$ level } & \multicolumn{4}{|c|}{$(2 R)-\mathbf{1}$ at BHandHLYP/6-31G* level } \\
\hline $\mathrm{C}$ & -1.071029 & -0.659109 & 0.031272 & $\mathrm{C}$ & -1.222190 & 0.085082 & 4885 \\
\hline $\mathrm{C}$ & -1.661912 & 0.641497 & -0.026539 & $\mathrm{C}$ & -1.223433 & 0.014708 & 1.258744 \\
\hline $\mathrm{C}$ & -0.838555 & 1.821330 & -0.207892 & $\mathrm{C}$ & 0.022069 & 0.033484 & 0109 \\
\hline $\mathrm{C}$ & 0.664777 & 1.574163 & -0.362302 & $\mathrm{C}$ & 1.268491 & 0.160221 & 1.152743 \\
\hline $\mathrm{C}$ & 1.104699 & 0.317253 & 0.419949 & $\mathrm{C}$ & 1.071782 & -0.475665 & -0.215620 \\
\hline $\mathrm{O}$ & 0.292831 & -0.839352 & 0.014584 & $\mathrm{O}$ & -0.074035 & 0.085577 & -0.849527 \\
\hline $\mathrm{C}$ & -1.859570 & -1.811890 & 0.092891 & $\mathrm{C}$ & -2.396564 & 0.169531 & -0.859878 \\
\hline $\mathrm{C}$ & -3.267994 & -1.673137 & 0.105775 & $\mathrm{C}$ & -3.603630 & 0.178749 & -0.163368 \\
\hline $\mathrm{C}$ & -3.895690 & -0.414508 & 0.069076 & $\mathrm{C}$ & -3.653351 & 0.097067 & 1.219422 \\
\hline $\mathrm{C}$ & -3.101279 & 0.740259 & 0.001384 & $\mathrm{C}$ & -2.470580 & 0.017822 & 1.930493 \\
\hline $\mathrm{C}$ & 2.566008 & -0.046835 & 0.213794 & $\mathrm{C}$ & 2.255962 & -0.286707 & -1.122256 \\
\hline $\mathrm{C}$ & 3.522607 & 0.312754 & 1.184578 & $\mathrm{C}$ & 3.261769 & -1.243658 & -1.145575 \\
\hline $\mathrm{C}$ & 4.885994 & 0.037730 & 0.981127 & $\mathrm{C}$ & 4.389979 & -1.060041 & -1.928497 \\
\hline
\end{tabular}




\begin{tabular}{|r|r|r|r||r|r|r|r|}
\hline $\mathrm{C}$ & 5.304835 & -0.612329 & -0.192437 & $\mathrm{C}$ & 4.517115 & 0.081499 & -2.703218 \\
$\mathrm{C}$ & 4.354333 & -0.983989 & -1.160839 & $\mathrm{C}$ & 3.512227 & 1.036525 & -2.689657 \\
$\mathrm{C}$ & 2.993597 & -0.699451 & -0.962323 & $\mathrm{C}$ & 2.388097 & 0.856334 & -1.900904 \\
$\mathrm{O}$ & -1.306995 & 2.989017 & -0.277653 & $\mathrm{O}$ & 0.086003 & -0.015639 & 3.211130 \\
$\mathrm{O}$ & -4.092728 & -2.770377 & 0.172077 & $\mathrm{O}$ & -4.779085 & 0.255163 & -0.810284 \\
$\mathrm{O}$ & -3.703480 & 1.949268 & -0.054923 & $\mathrm{O}$ & -2.528776 & -0.043102 & 3.254636 \\
$\mathrm{H}$ & 1.218328 & 2.461511 & -0.016817 & $\mathrm{H}$ & 2.101560 & -0.292892 & 1.679874 \\
$\mathrm{H}$ & 0.895175 & 1.441927 & -1.436451 & $\mathrm{H}$ & 1.492896 & 1.220059 & 1.030577 \\
$\mathrm{H}$ & 0.911599 & 0.485775 & 1.497008 & $\mathrm{H}$ & 0.884856 & -1.542043 & -0.083317 \\
$\mathrm{H}$ & -1.379115 & -2.791998 & 0.152370 & $\mathrm{H}$ & -2.361404 & 0.209527 & -1.935736 \\
$\mathrm{H}$ & -4.983070 & -0.336296 & 0.084200 & $\mathrm{H}$ & -4.597023 & 0.102498 & 1.733676 \\
$\mathrm{H}$ & 3.199226 & 0.809378 & 2.105542 & $\mathrm{H}$ & 3.161999 & -2.139766 & -0.552095 \\
$\mathrm{H}$ & 5.617067 & 0.323016 & 1.743296 & $\mathrm{H}$ & 5.163029 & -1.811145 & -1.938909 \\
$\mathrm{H}$ & 6.364628 & -0.833354 & -0.349751 & $\mathrm{H}$ & 5.391089 & 0.223917 & -3.317849 \\
$\mathrm{H}$ & 4.673024 & -1.497694 & -2.072760 & $\mathrm{H}$ & 3.602693 & 1.923601 & -3.295544 \\
$\mathrm{H}$ & 2.254757 & -1.000314 & -1.709443 & $\mathrm{H}$ & 1.602529 & 1.592889 & -1.899067 \\
$\mathrm{H}$ & -3.535149 & -3.578158 & 0.184161 & $\mathrm{H}$ & -4.630861 & 0.312655 & -1.754819 \\
$\mathrm{H}$ & -2.954737 & 2.627723 & -0.134520 & $\mathrm{H}$ & -1.615874 & -0.064101 & 3.598300 \\
\hline
\end{tabular}

TABLE S2. Optimized Z-Matrixes of Conformers of $(2 R, 3 S)-2$ in the Gas Phase $(\AA)$

\begin{tabular}{|l|r|r|r||l||l|r|r|r|}
\hline \multicolumn{2}{|l|}{$\mathbf{2 a 1}$} & \multicolumn{3}{|l|}{ 2a2 } \\
\hline C & 1.418159 & -0.525328 & -0.388033 & C & 1.472197 & -0.585584 & -0.317315 \\
C & 2.670371 & -0.318034 & -1.240836 & C & 2.815989 & -0.486539 & -1.037442 \\
C & 3.672233 & 0.560648 & -0.698894 & C & 3.754187 & 0.464855 & -0.505596 \\
C & 4.783483 & 1.002169 & -1.488384 & C & 4.955233 & 0.802629 & -1.210937 \\
C & 5.681759 & 1.936484 & -0.982164 & C & 5.795049 & 1.804718 & -0.735298 \\
C & 5.498567 & 2.423649 & 0.314571 & C & 5.462896 & 2.465024 & 0.450631 \\
C & 4.442104 & 1.987748 & 1.132025 & C & 4.313531 & 2.137322 & 1.189199 \\
C & 3.546046 & 1.059885 & 0.623265 & C & 3.476143 & 1.140484 & 0.711496 \\
O & 2.548906 & 0.646136 & 1.442146 & O & 2.386090 & 0.836272 & 1.455891 \\
C & 1.872532 & -0.590317 & 1.102229 & C & 1.742603 & -0.442047 & 1.209889 \\
C & 0.761447 & -0.779384 & 2.106009 & C & 0.516564 & -0.496058 & 2.085985 \\
C & 0.427314 & -2.063052 & 2.548102 & C & 0.218476 & -1.656398 & 2.805058 \\
C & -0.621816 & -2.263891 & 3.443595 & C & -0.928250 & -1.738572 & 3.594035 \\
C & -1.348649 & -1.169070 & 3.921696 & C & -1.791204 & -0.642357 & 3.680521 \\
C & -1.013548 & 0.123664 & 3.502324 & C & -1.498412 & 0.531778 & 2.975576 \\
C & 0.030164 & 0.308978 & 2.602127 & C & -0.357032 & 0.596636 & 2.185305 \\
C & 0.521390 & -1.677165 & -0.793984 & C & 0.607973 & -1.783067 & -0.660331 \\
C & 0.992282 & -2.992580 & -1.004829 & C & 1.077709 & -3.116173 & -0.667491 \\
C & 0.133988 & -4.078154 & -1.187841 & C & 0.221009 & -4.212370 & -0.788985 \\
C & -1.244504 & -3.883981 & -1.209628 & C & -1.148979 & -4.018761 & -0.941721 \\
C & -1.767005 & -2.567981 & -1.071246 & C & -1.665823 & -2.694372 & -0.997265 \\
C & -3.201261 & -2.318858 & -1.127446 & C & -3.090389 & -2.447959 & -1.176349 \\
C & -3.595612 & -0.936140 & -0.976110 & C & -3.479252 & -1.054452 & -1.205507 \\
C & -2.676609 & 0.050343 & -0.800526 & C & -2.565777 & -0.060039 & -1.051424 \\
O & -1.343098 & -0.223956 & -0.727712 & O & -1.239656 & -0.329070 & -0.891783 \\
\hline
\end{tabular}




\begin{tabular}{|c|c|c|c|c|c|c|c|}
\hline $\mathrm{C}$ & -0.867528 & -1.507588 & -0.867209 & $\mathrm{C}$ & -0.768989 & -1.621433 & -0.861012 \\
\hline $\mathrm{C}$ & -2.966558 & 1.484543 & -0.667692 & $\mathrm{C}$ & -2.849853 & 1.381716 & -1.047761 \\
\hline $\mathrm{C}$ & -4.172008 & 2.012991 & -1.172349 & $\mathrm{C}$ & -4.127467 & 1.850027 & 915 \\
\hline $\mathrm{C}$ & -4.456402 & 3.360867 & -1.033894 & $\mathrm{C}$ & -4.404761 & 3.206789 & -0.696509 \\
\hline $\mathrm{C}$ & -3.545364 & 4.225041 & -0.394411 & $\mathrm{C}$ & -3.415438 & 4.137870 & -1.070476 \\
\hline $\mathrm{C}$ & -2.349935 & 3.708341 & 0.098525 & $\mathrm{C}$ & -2.149465 & 3.679334 & -1.426535 \\
\hline $\mathrm{C}$ & -2.061407 & 2.352454 & -0.039407 & $\mathrm{C}$ & -1.867527 & 2.314908 & 288 \\
\hline $\mathrm{O}$ & 2.798081 & -0.875644 & -2.354617 & $\mathrm{O}$ & 3.067314 & -1.185559 & 253 \\
\hline $\mathrm{O}$ & 4.966500 & 0.544058 & -2.732574 & $\mathrm{O}$ & 5.280938 & 0.179462 & 19883 \\
\hline $\mathrm{O}$ & 6.406432 & 3.327248 & 698 & $\mathrm{O}$ & 6.318639 & 3.429214 & 1128 \\
\hline $\mathrm{O}$ & -2.384157 & -1.295331 & 4.802851 & $\mathrm{O}$ & -2.928634 & -0.653273 & 7246 \\
\hline $\mathrm{O}$ & 2.332536 & -3.262999 & -1.00 & $\mathrm{O}$ & 2.407063 & 0792 & 853 \\
\hline $\mathrm{O}$ & -2.061274 & -4.929237 & -1.387831 & $\mathrm{O}$ & -1.961980 & -5.075763 & -1.059090 \\
\hline $\mathrm{O}$ & -4.034001 & -3.240218 & -1.289193 & $\mathrm{O}$ & -3.919549 & -3.378053 & 9755 \\
\hline $\mathrm{O}$ & -5.596102 & 3.974120 & -1.498589 & $\mathrm{O}$ & -5.611628 & 3.764681 & 6155 \\
\hline $\mathrm{O}$ & -3.826006 & 5.546209 & -0.265981 & $\mathrm{O}$ & -3.689792 & 5.466747 & 214 \\
\hline $\mathrm{H}$ & 0.84 & 0.403833 & -0.4 & $\mathrm{H}$ & 0.924082 & 0.319503 & -0 . \\
\hline $\mathrm{H}$ & 4.240521 & -0.106505 & -2.914990 & $\mathrm{H}$ & 4.576819 & -0.496273 & 702 \\
\hline $\mathrm{H}$ & 6.51 & 2.278000 & -1.5 & $\mathrm{H}$ & 6.695844 & 2.066080 & -1. \\
\hline $\mathrm{H}$ & 6.180257 & 3.592087 & 1.665495 & $\mathrm{H}$ & 5.987205 & 3.816235 & 7065 \\
\hline $\mathrm{H}$ & 4.32 & 2.335290 & 2.154494 & $\mathrm{H}$ & 4.083134 & 2.622220 & 933 \\
\hline $\mathrm{H}$ & 2.598097 & -1.409117 & 1.199141 & $\mathrm{H}$ & 2.442335 & -1.233824 & 138 \\
\hline $\mathrm{H}$ & 0.986059 & -2.922369 & 2.187049 & $\mathrm{H}$ & 0.884008 & -2.513870 & 687 \\
\hline $\mathrm{H}$ & -0.869610 & -3.270856 & 796 & $\mathrm{H}$ & -1.147253 & -2.652201 & 3421 \\
\hline $\mathrm{H}$ & -2.520401 & -2.235368 & 9511 & $\mathrm{H}$ & -3.016299 & -1.5 & 321 \\
\hline $\mathrm{H}$ & -1.57 & 0.965234 & 494 & $\mathrm{H}$ & -2.174340 & 1.376902 & 839 \\
\hline $\mathrm{H}$ & 0.299395 & 1.315660 & 2.298431 & $\mathrm{H}$ & -0.135911 & 1.512477 & 121 \\
\hline $\mathrm{H}$ & 2.75 & -2.593504 & -1.5 & $\mathrm{H}$ & 2.892319 & -2.783594 & 766 \\
\hline $\mathrm{H}$ & 0.549900 & -5.066336 & -1.344704 & $\mathrm{H}$ & 0.635052 & -5.213702 & 1395 \\
\hline $\mathrm{H}$ & -2.991185 & -4.560575 & -1.39 & $\mathrm{H}$ & -2.884123 & -4.708800 & 0289 \\
\hline $\mathrm{H}$ & -4.65 & -0.710897 & -0.983038 & $\mathrm{H}$ & -4.523195 & -0.824547 & 828 \\
\hline $\mathrm{H}$ & -4.874079 & 1.371263 & -1.698202 & $\mathrm{H}$ & -4.893766 & 1.150447 & 148 \\
\hline $\mathrm{H}$ & -6.16 & 3.325706 & -1.93 & $\mathrm{H}$ & 3645 & 3.06 & -0. \\
\hline $\mathrm{H}$ & -4.695312 & 5.705948 & -0.6 & $\mathrm{H}$ & 6996 & 5.579827 & 619 \\
\hline $\mathrm{H}$ & -1.656854 & 4.383312 & 123 & $\mathrm{H}$ & -1.3 & 413 & 726 \\
\hline $\mathrm{H}$ & -1.136911 & 1.958425 & 0.363772 & $\mathrm{H}$ & -0. & 1.970126 & -1.709106 \\
\hline \multicolumn{4}{|c|}{$2 \mathrm{a} 3$} & \multicolumn{4}{|c|}{$2 a 4$} \\
\hline $\mathrm{C}$ & 1.217652 & 092 & 50654 & $\mathrm{C}$ & 1.238892 & -0.732676 & -0.339370 \\
\hline $\mathrm{C}$ & 2.521516 & -0.784002 & -1.148518 & $\mathrm{C}$ & 2.572688 & -0.735053 & -1.084092 \\
\hline $\mathrm{C}$ & 3.620174 & -0.021230 & -0.616766 & $\mathrm{C}$ & 3.646650 & 0.004554 & 6714 \\
\hline $\mathrm{C}$ & 4.820879 & 0.182555 & -1.371757 & $\mathrm{C}$ & 4.877773 & 0.238225 & -1.171888 \\
\hline $\mathrm{C}$ & 5.831341 & 1.005555 & -0.882946 & $\mathrm{C}$ & 5.867490 & 1.036598 & -0.607419 \\
\hline $\mathrm{C}$ & 5.670365 & 1.613824 & 0.364253 & $\mathrm{C}$ & 5.656857 & 1.589761 & 0.658467 \\
\hline $\mathrm{C}$ & 4.521662 & 1.411873 & 1.148594 & $\mathrm{C}$ & 4.474299 & 1.362230 & 1.382394 \\
\hline $\mathrm{C}$ & 3.514670 & 0.595468 & 0.656486 & $\mathrm{C}$ & 3.487397 & 0.568957 & 0.816187 \\
\hline $\mathrm{O}$ & 2.425619 & 0.410245 & 1.442181 & $\mathrm{O}$ & 2.367779 & 0.354089 & 1.547393 \\
\hline $\mathrm{C}$ & 1.589840 & -0.742079 & 1.163710 & $\mathrm{C}$ & 1.542361 & -0.785899 & 1.187857 \\
\hline $\mathrm{C}$ & 0.419131 & -0.681423 & 2.114509 & $\mathrm{C}$ & 0.328443 & -0.752524 & 2.081147 \\
\hline
\end{tabular}




\begin{tabular}{|c|c|c|c|c|c|c|c|}
\hline $\mathrm{C}$ & 29319 & -1.861257 & 2.626711 & $\mathrm{C}$ & -0.125912 & -1.923325 & 2.693769 \\
\hline $\mathrm{C}$ & -1.234641 & -1.830809 & 3.475034 & $\mathrm{C}$ & -1.267763 & -1.919455 & 3.493913 \\
\hline $\mathrm{C}$ & -1.803297 & -0.604872 & 3.835135 & $\mathrm{C}$ & -1.967414 & -0.726812 & 3.699745 \\
\hline $\mathrm{C}$ & -1.254726 & 0.585652 & 3.343970 & $\mathrm{C}$ & -1.516331 & 0.457198 & 3.102631 \\
\hline $\mathrm{C}$ & -0.156979 & 0.539445 & 2.491679 & $\mathrm{C}$ & -0.381730 & 0.436974 & 0708 \\
\hline $\mathrm{C}$ & 0.18 & -1.796187 & -0.708083 & $\mathrm{C}$ & 0.225227 & -1.773078 & -0.7 \\
\hline $\mathrm{C}$ & 0.481752 & -3.175774 & -0.787550 & $\mathrm{C}$ & 0.524061 & 8008 & -0.9 \\
\hline $\mathrm{C}$ & -0.509106 & 9769 & -0.917453 & $\mathrm{C}$ & -0.464972 & 5929 & -1.0 \\
\hline $\mathrm{C}$ & -1.847807 & -3.777011 & -1.011474 & $\mathrm{C}$ & -1.803343 & -3.742331 & -1.180265 \\
\hline $\mathrm{C}$ & -2.190646 & -2.396324 & -1.003800 & $\mathrm{C}$ & -2.150492 & -2.364246 & 533 \\
\hline $\mathrm{C}$ & -3.576958 & -1.965425 & -1.130347 & $\mathrm{C}$ & -3.536960 & -1.929837 & -1.2 \\
\hline $\mathrm{C}$ & -3.787024 & -0.535538 & -1.108422 & $\mathrm{C}$ & -3.748629 & -0.500591 & -1 . \\
\hline $\mathrm{C}$ & -2.748708 & 0.333778 & -0.986433 & $\mathrm{C}$ & -2.713386 & 0.357882 & -0.9 \\
\hline $\mathrm{O}$ & -1.465280 & -0.108200 & -0.841180 & $\mathrm{O}$ & -1.427550 & 5516 & -0.8 \\
\hline $\mathrm{C}$ & -1.1 & -1.4 & -0.8 & $\mathrm{C}$ & -1 . & -1. & -0 \\
\hline $\mathrm{C}$ & -2.843300 & 1.799832 & -0.992878 & $\mathrm{C}$ & -2.8 & 505 & -0 . \\
\hline $\mathrm{C}$ & -3.9 & 544 & -1.5 & $\mathrm{C}$ & & 71 & -1 . \\
\hline $\mathrm{C}$ & -4.068987 & 3.835050 & -1.516480 & $\mathrm{C}$ & -1.8 & 803 & -1. \\
\hline $\mathrm{C}$ & -3.0 & 346 & -1.003280 & $\mathrm{C}$ & & 45 & -0 . \\
\hline $\mathrm{C}$ & -1.888084 & 3.969019 & -0.486290 & $\mathrm{C}$ & -4.090549 & 791 & -0.3 \\
\hline $\mathrm{C}$ & -1.7 & 128 & -0.480 & $\mathrm{C}$ & -3.9 & 34 & -0 . \\
\hline $\mathrm{O}$ & 2.611749 & -1.434528 & -2.214089 & $\mathrm{O}$ & 2.705796 & -1.336622 & -2.1 \\
\hline $\mathrm{O}$ & 4.9 & -0.3 & -2.567575 & $\mathrm{O}$ & 80 & 42 & -2 . \\
\hline $\mathrm{O}$ & 6.68 & 947 & 0.796 & $\mathrm{O}$ & 6.6 & 204 & 242 \\
\hline $\mathrm{O}$ & -2.88 & -0.5 & 4.6 & $\mathrm{O}$ & -3.0 & -0. & 4. \\
\hline $\mathrm{O}$ & 1.7 & -3.6 & -0.698477 & $\mathrm{O}$ & 540 & 004 & -0.8 \\
\hline $\mathrm{O}$ & -2.792996 & -4.715786 & -1.134346 & $\mathrm{O}$ & -2.745098 & -4.677227 & -1.3 \\
\hline $\mathrm{O}$ & -4.520362 & -2.78 & -1.243833 & $\mathrm{O}$ & -4.477445 & 947 & -1.3 \\
\hline $\mathrm{O}$ & -3.11 & 178 & -1.009136 & $\mathrm{O}$ & -0.817 & 4.892209 & -1. \\
\hline $\mathrm{O}$ & -0.92 & 4.822062 & -0.002291 & $\mathrm{O}$ & -3.1 & 368 & -0.6 \\
\hline $\mathrm{H}$ & 0.78 & 0.240032 & -0.561366 & $\mathrm{H}$ & 0.80 & 859 & -0.5 \\
\hline $\mathrm{H}$ & 4.175975 & -0.938625 & -2.744849 & $\mathrm{H}$ & 528 & 315 & $-2 .($ \\
\hline $\mathrm{H}$ & 6.733185 & 1.165674 & -1.461040 & $\mathrm{H}$ & 6.793482 & 1.216326 & -1.1 \\
\hline $\mathrm{H}$ & 6.473295 & 2.755008 & 1.672014 & $\mathrm{H}$ & 6.404793 & 2135 & 498 \\
\hline $\mathrm{H}$ & 4.420844 & 1.850057 & 2.136957 & $\mathrm{H}$ & 4.330292 & 1.761002 & 961 \\
\hline $\mathrm{H}$ & 2.181610 & -1.644625 & 1.367200 & $\mathrm{H}$ & 2.121795 & -1.696969 & 7862 \\
\hline $\mathrm{H}$ & 0.304610 & -2.820603 & 2.357918 & $\mathrm{H}$ & 0.410687 & -2.856190 & 025 \\
\hline $\mathrm{H}$ & -1.649410 & -2.760034 & 3.860857 & $\mathrm{H}$ & -1.609852 & -2.841722 & 476 \\
\hline $\mathrm{H}$ & -3.175505 & -1.393115 & 4.916006 & $\mathrm{H}$ & -3.304724 & -1.534735 & 4.809075 \\
\hline $\mathrm{H}$ & -1.698874 & 1.530161 & 3.641646 & $\mathrm{H}$ & -2.066866 & 1.376154 & 3.276468 \\
\hline $\mathrm{H}$ & 0.278806 & 985 & 2.133745 & $\mathrm{H}$ & -0.035865 & 870 & 443 \\
\hline $\mathrm{H}$ & 2.305501 & -3.071843 & -1.309894 & $\mathrm{H}$ & 2.357856 & -3.004474 & -1.380278 \\
\hline $\mathrm{H}$ & -0.226564 & -5.194364 & -0.973792 & $\mathrm{H}$ & -0.180399 & -5.156837 & -1.188495 \\
\hline $\mathrm{H}$ & -3.664493 & -4.228502 & -1.207805 & $\mathrm{H}$ & -3.616626 & -4.190169 & -1.413839 \\
\hline $\mathrm{H}$ & -4.805206 & -0.175560 & -1.178646 & $\mathrm{H}$ & -4.757933 & -0.128691 & -1.264448 \\
\hline $\mathrm{H}$ & -4.782400 & 1.865634 & -1.947176 & $\mathrm{H}$ & -0.797624 & 2.177182 & -1.565522 \\
\hline $\mathrm{H}$ & -4.938415 & 4.338203 & -1.926732 & $\mathrm{H}$ & -0.049404 & 4.407104 & -1.813485 \\
\hline $\mathrm{H}$ & -2.295936 & 6.315300 & -0.625579 & $\mathrm{H}$ & -2.260161 & 6.347555 & -0.931621 \\
\hline
\end{tabular}




\begin{tabular}{|c|c|c|c|c|c|c|c|}
\hline $\mathrm{H}$ & -0.147540 & 4.316000 & 0.283888 & $\mathrm{H}$ & -4.998599 & 4.302025 & 0.017104 \\
\hline $\mathrm{H}$ & -0.914681 & 2.108634 & -0.060452 & $\mathrm{H}$ & -4.832694 & 1.826166 & -0.092240 \\
\hline \multicolumn{4}{|c|}{$2 \mathrm{a} 5$} & \multicolumn{4}{|c|}{$2 \mathrm{a} 6$} \\
\hline $\mathrm{C}$ & -1.352828 & 0.379080 & -0.225049 & $\mathrm{C}$ & -1.375633 & 0.400979 & -0.227386 \\
\hline $\mathrm{C}$ & -2.423094 & 0.177159 & -1.310105 & $\mathrm{C}$ & -2.512495 & 0.178902 & -1.235586 \\
\hline $\mathrm{C}$ & -3.553549 & -0.653870 & -0.955013 & $\mathrm{C}$ & -3.612382 & -0.648861 & -0.787786 \\
\hline $\mathrm{C}$ & -4.550497 & -0.991802 & -1.922812 & $\mathrm{C}$ & -4.665071 & -1.018950 & -1.682131 \\
\hline $\mathrm{C}$ & -5.586809 & -1.864936 & -1.598443 & $\mathrm{C}$ & -5.668470 & -1.894680 & -1.272979 \\
\hline $\mathrm{C}$ & -5.633475 & -2.418085 & -0.317395 & $\mathrm{C}$ & -5.627619 & -2.417741 & 0.020961 \\
\hline $\mathrm{C}$ & -4.674285 & -2.108182 & 0.659927 & $\mathrm{C}$ & -4.612859 & -2.074776 & 0.928482 \\
\hline $\mathrm{C}$ & -3.650128 & -1.229738 & 0.333731 & $\mathrm{C}$ & -3.620853 & -1.194170 & 0.518673 \\
\hline $\mathrm{O}$ & -2.729034 & -0.967950 & 1.297946 & $\mathrm{O}$ & -2.645727 & -0.898324 & 1.417694 \\
\hline $\mathrm{C}$ & -1.996323 & 0.274899 & 1.184519 & $\mathrm{C}$ & -1.932916 & 0.348160 & 1.219294 \\
\hline $\mathrm{C}$ & -0.996124 & 0.305699 & 2.315293 & $\mathrm{C}$ & -0.869949 & 0.431292 & 2.286528 \\
\hline $\mathrm{C}$ & -0.714503 & 1.504587 & 2.975499 & $\mathrm{C}$ & -0.697813 & 1.605982 & 3.022433 \\
\hline $\mathrm{C}$ & 0.239961 & 1.560610 & 3.990929 & $\mathrm{C}$ & 0.305129 & 1.712180 & 3.986096 \\
\hline $\mathrm{C}$ & 0.922427 & 0.400631 & 4.368753 & $\mathrm{C}$ & 1.148415 & 0.625190 & 4.232064 \\
\hline $\mathrm{C}$ & 0.640416 & -0.811301 & 3.727080 & $\mathrm{C}$ & 0.981188 & -0.563216 & 3.510426 \\
\hline $\mathrm{C}$ & -0.307729 & -0.850751 & 2.710101 & $\mathrm{C}$ & -0.016433 & -0.651100 & 2.546842 \\
\hline $\mathrm{C}$ & -0.552714 & 1.643669 & -0.443497 & $\mathrm{C}$ & -0.565159 & 1.641764 & -0.523424 \\
\hline $\mathrm{C}$ & -1.167261 & 2.908165 & -0.504614 & $\mathrm{C}$ & 276 & 157 & 2367 \\
\hline $\mathrm{C}$ & -0.461496 & 4.086901 & -0.755754 & $\mathrm{C}$ & -0.422850 & 4.080481 & -0.844334 \\
\hline $\mathrm{C}$ & 0.914132 & 4.038711 & -0.970342 & $\mathrm{C}$ & 0.943626 & 3.997235 & -1.102519 \\
\hline $\mathrm{C}$ & 1.584641 & 2.786869 & -0.920644 & $\mathrm{C}$ & 1.583047 & 2.728633 & -1.075410 \\
\hline $\mathrm{C}$ & 3.020569 & 2.697060 & -1.144729 & $\mathrm{C}$ & 3.008571 & 2.600928 & -1.341671 \\
\hline $\mathrm{C}$ & 3.574925 & 1.364102 & -1.068693 & $\mathrm{C}$ & 3.525353 & 1.250846 & -1.304509 \\
\hline $\mathrm{C}$ & 2.789524 & 0.281995 & -0.820531 & $\mathrm{C}$ & 2.723085 & 0.190795 & -1.017653 \\
\hline $\mathrm{O}$ & 1.447780 & 0.405139 & -0.613497 & $\mathrm{O}$ & 1.392472 & 0.350253 & -0.769948 \\
\hline $\mathrm{C}$ & 0.828826 & 1.630627 & -0.655167 & $\mathrm{C}$ & 0.804538 & 1.591625 & -0.794670 \\
\hline $\mathrm{C}$ & 3.246523 & -1.110379 & -0.726381 & $\mathrm{C}$ & 3.141251 & -1.215505 & -0.946029 \\
\hline $\mathrm{C}$ & 4.410451 & -1.520694 & -1.406528 & $\mathrm{C}$ & 4.487973 & -1.542489 & -0.690018 \\
\hline $\mathrm{C}$ & 4.856036 & -2.827350 & -1.299617 & $\mathrm{C}$ & 4.888264 & -2.867057 & -0.642562 \\
\hline $\mathrm{C}$ & 4.152495 & -3.763430 & -0.515740 & $\mathrm{C}$ & 3.957442 & -3.905867 & -0.843141 \\
\hline $\mathrm{C}$ & 2.999388 & -3.361553 & 0.153980 & $\mathrm{C}$ & 2.624468 & -3.586956 & -1.090204 \\
\hline $\mathrm{C}$ & 2.546869 & -2.048348 & 0.047414 & $\mathrm{C}$ & 2.218679 & -2.255137 & -1.137926 \\
\hline $\mathrm{O}$ & -2.274159 & 0.660708 & -2.442522 & $\mathrm{O}$ & -2.440886 & 0.639749 & -2.384979 \\
\hline $\mathrm{O}$ & -4.500302 & -0.484229 & -3.160959 & $\mathrm{O}$ & -4.698793 & -0.540469 & -2.932588 \\
\hline $\mathrm{O}$ & -6.662210 & -3.266180 & -0.052514 & $\mathrm{O}$ & -6.627694 & -3.269932 & 0.369558 \\
\hline $\mathrm{O}$ & 1.864373 & 0.383592 & 5.358524 & $\mathrm{O}$ & 2.148113 & 0.658889 & 5.163819 \\
\hline $\mathrm{O}$ & -2.514233 & 2.936741 & -0.283526 & $\mathrm{O}$ & -2.484241 & 2.985149 & -0.285005 \\
\hline $\mathrm{O}$ & 1.587349 & 5.168699 & -1.212758 & $\mathrm{O}$ & 1.637869 & 5.109931 & -1.365841 \\
\hline $\mathrm{O}$ & 3.724010 & 3.707650 & -1.376613 & $\mathrm{O}$ & 3.731439 & 3.592153 & -1.595796 \\
\hline $\mathrm{O}$ & 5.969154 & -3.331607 & -1.930632 & $\mathrm{O}$ & 6.171699 & -3.290419 & -0.388210 \\
\hline $\mathrm{O}$ & 4.590355 & -5.044019 & -0.418357 & $\mathrm{O}$ & 4.353223 & -5.202433 & -0.789531 \\
\hline $\mathrm{H}$ & -0.671847 & -0.475664 & -0.324431 & $\mathrm{H}$ & -0.715931 & -0.469071 & -0.334856 \\
\hline $\mathrm{H}$ & -6.338508 & -2.118772 & -2.335921 & $\mathrm{H}$ & -6.462380 & -2.173249 & -1.955202 \\
\hline $\mathrm{H}$ & -4.723839 & -2.523220 & 1.662164 & $\mathrm{H}$ & -4.594898 & -2.465974 & 1.941307 \\
\hline $\mathrm{H}$ & -2.713787 & 1.094949 & 1.299833 & $\mathrm{H}$ & -2.651917 & 1.164817 & 1.346966 \\
\hline
\end{tabular}




\begin{tabular}{|c|c|c|c|c|c|c|c|}
\hline $\mathrm{H}$ & -1.244793 & 2.411096 & 2.695587 & $\mathrm{H}$ & -1.351947 & 2.455049 & 068 \\
\hline $\mathrm{H}$ & 0.446818 & 2.504004 & 4.492963 & $\mathrm{H}$ & 0.426191 & 2.636117 & 4.548745 \\
\hline $\mathrm{H}$ & 1.168020 & -1.706276 & 4.041489 & $\mathrm{H}$ & 1.639111 & -1.400966 & 3.718676 \\
\hline $\mathrm{H}$ & -0.539094 & -1.797306 & 2.230895 & $\mathrm{H}$ & -0.146459 & -1.579727 & 1.999081 \\
\hline $\mathrm{H}$ & -0.967785 & 5.047553 & -0.790108 & $\mathrm{H}$ & -0.904806 & 5.054046 & -0.861750 \\
\hline $\mathrm{H}$ & 4.645602 & 1.253084 & -1.184317 & $\mathrm{H}$ & 4.571132 & 1.104290 & 2621 \\
\hline $\mathrm{H}$ & 4.947891 & -0.821409 & -2.042005 & $\mathrm{H}$ & 5.215073 & -0.757627 & -0.498843 \\
\hline $\mathrm{H}$ & 2.470025 & -4.091203 & 0.758094 & $\mathrm{H}$ & 1.917834 & -4.395252 & -1.247710 \\
\hline $\mathrm{H}$ & 1.661268 & -1.739800 & 0.590392 & $\mathrm{H}$ & 1.181880 & -2.020232 & -1.347426 \\
\hline $\mathrm{H}$ & -3.677193 & 0.073350 & -3.207316 & $\mathrm{H}$ & -3.887598 & 0.024990 & -3.044328 \\
\hline $\mathrm{H}$ & -6.582514 & -3.587738 & 0.859387 & $\mathrm{H}$ & -6.487750 & -3.568130 & 1.282139 \\
\hline $\mathrm{H}$ & 1.967441 & 1.280532 & 5.712949 & $\mathrm{H}$ & 2.156738 & 1.532007 & 5.586095 \\
\hline $\mathrm{H}$ & -2.851499 & 3.826515 & -0.472423 & $\mathrm{H}$ & -2.802012 & 3.887441 & -0.4 \\
\hline $\mathrm{H}$ & 2.545574 & 4.900862 & -1.334300 & $\mathrm{H}$ & 2.584349 & 4.817843 & -1.517388 \\
\hline $\mathrm{H}$ & 6.398394 & -2.637718 & -2.453444 & $\mathrm{H}$ & 6.756543 & -2.526938 & -0.2 \\
\hline $\mathrm{H}$ & 5.396972 & -5.123242 & -0.95 & $\mathrm{H}$ & 526 & -5.213678 & 720 \\
\hline \multicolumn{4}{|c|}{$2 a 7$} & \multicolumn{4}{|c|}{$2 \mathrm{a} 8$} \\
\hline $\mathrm{C}$ & -1.241073 & 0.516559 & -0.12 & $\mathrm{C}$ & 1.161091 & -0.579719 & -0.2 \\
\hline $\mathrm{C}$ & -2.407331 & 0.574973 & -1.147471 & $\mathrm{C}$ & 2.303871 & -0.347429 & -1.2 \\
\hline $\mathrm{C}$ & -3.559557 & -0.248487 & -0.849184 & $\mathrm{C}$ & 3.490926 & 0.290191 & -0.7 \\
\hline $\mathrm{C}$ & -4.652350 & -0.344618 & -1.766649 & $\mathrm{C}$ & 4.564407 & 0.677565 & -1.600217 \\
\hline $\mathrm{C}$ & -5.719252 & -1.203831 & -1.510693 & $\mathrm{C}$ & 5.658034 & 1.380406 & -1.05 \\
\hline $\mathrm{C}$ & -5.703994 & -1.980322 & -0.350850 & $\mathrm{C}$ & 5.689555 & 1.711288 & 0.257158 \\
\hline $\mathrm{C}$ & -4.649197 & -1.911734 & 0.573946 & $\mathrm{C}$ & 4.657470 & 1.344010 & 1.1 \\
\hline $\mathrm{C}$ & -3.594025 & -1.047585 & 0.317263 & $\mathrm{C}$ & 3.573845 & 0.635942 & 0.632529 \\
\hline $\mathrm{O}$ & -2.578711 & -1.025069 & 1.221835 & $\mathrm{O}$ & 2.587623 & 0.309638 & 069 \\
\hline $\mathrm{C}$ & -1.769879 & 0.172576 & 1.273287 & $\mathrm{C}$ & 1.744640 & -0.814499 & 1.146177 \\
\hline $\mathrm{C}$ & -0.676277 & -0.078023 & 2.286060 & $\mathrm{C}$ & 0.700808 & -0.960205 & 2.224643 \\
\hline $\mathrm{C}$ & -0.188822 & 0.967654 & 3.075010 & $\mathrm{C}$ & 0.459445 & -2.205128 & 2.810114 \\
\hline $\mathrm{C}$ & 0.850960 & 0.762430 & 3.981490 & $\mathrm{C}$ & -0.525908 & -2.364563 & 3.784457 \\
\hline $\mathrm{C}$ & 1.413652 & -0.509760 & 4.120373 & $\mathrm{C}$ & -1.281723 & -1.262302 & 4.193459 \\
\hline $\mathrm{C}$ & 0.923624 & -1.571795 & 3.351055 & $\mathrm{C}$ & -1.044876 & -0.005229 & 3.622940 \\
\hline $\mathrm{C}$ & -0.107733 & -1.350340 & 2.443672 & $\mathrm{C}$ & -0.065226 & 36214 & 2.647739 \\
\hline $\mathrm{C}$ & -0.388288 & 1.764677 & -0.196462 & $\mathrm{C}$ & 0.188355 & -1.646065 & -0.718623 \\
\hline $\mathrm{C}$ & -0.937725 & 3.043108 & 0.014341 & $\mathrm{C}$ & 0.586853 & -2.975611 & -0.946340 \\
\hline $\mathrm{C}$ & -0.190199 & 4.218570 & -0.081922 & $\mathrm{C}$ & -0.299925 & -3.974878 & -1.353929 \\
\hline $\mathrm{C}$ & 1.162464 & 4.154418 & -0.410792 & $\mathrm{C}$ & -1.645835 & -3.671230 & -1.549740 \\
\hline $\mathrm{C}$ & 1.768023 & 2.887979 & -0.631454 & $\mathrm{C}$ & -2.099610 & -2.341487 & -1.338041 \\
\hline $\mathrm{C}$ & 3.178785 & 2.782954 & -0.980085 & $\mathrm{C}$ & -3.498252 & -1.984861 & -1.534009 \\
\hline $\mathrm{C}$ & 3.667659 & 1.437304 & -1.178677 & $\mathrm{C}$ & -3.818136 & -0.591688 & -1.312472 \\
\hline $\mathrm{C}$ & 2.847110 & 0.360136 & -1.056562 & $\mathrm{C}$ & -2.867180 & 0.301066 & -0.929693 \\
\hline $\mathrm{O}$ & 1.530352 & 0.496975 & -0.723344 & $\mathrm{O}$ & -1.568150 & -0.072790 & -0.743878 \\
\hline $\mathrm{C}$ & 0.973505 & 1.733983 & -0.511595 & $\mathrm{C}$ & -1.163671 & -1.370920 & -0.940617 \\
\hline $\mathrm{C}$ & 3.236670 & -1.043649 & -1.235736 & $\mathrm{C}$ & -3.074992 & 1.735419 & -0.692314 \\
\hline $\mathrm{C}$ & 4.313910 & -1.391190 & -2.065232 & $\mathrm{C}$ & -2.006731 & 2.641644 & -0.843544 \\
\hline $\mathrm{C}$ & 4.692090 & -2.721969 & -2.221424 & $\mathrm{C}$ & -2.209312 & 3.997481 & -0.644720 \\
\hline $\mathrm{C}$ & 4.000603 & -3.730071 & -1.552296 & $\mathrm{C}$ & -3.478581 & 4.487909 & -0.281177 \\
\hline $\mathrm{C}$ & 2.916593 & -3.386935 & -0.721548 & $\mathrm{C}$ & -4.534479 & 3.593010 & -0.120021 \\
\hline
\end{tabular}




\begin{tabular}{|c|c|c|c|c|c|c|c|}
\hline $\mathrm{C}$ & 2.534952 & -2.064807 & -0.565826 & $\mathrm{C}$ & -4.335164 & 2.229997 & -0.323450 \\
\hline $\mathrm{O}$ & -2.317224 & 1.254041 & -2.180884 & $\mathrm{O}$ & 2.168155 & -0.625989 & -2.470061 \\
\hline $\mathrm{O}$ & -4.665728 & 0.379484 & -2.892444 & $\mathrm{O}$ & 4.530907 & 0.384874 & -2.906845 \\
\hline $\mathrm{O}$ & -6.767146 & -2.802608 & -0.149164 & $\mathrm{O}$ & 6.777237 & 2.398927 & 0.694735 \\
\hline $\mathrm{O}$ & 2.428458 & -0.782528 & 4.992511 & $\mathrm{O}$ & -2.258445 & -1.344934 & 5.144988 \\
\hline $\mathrm{O}$ & -2.262050 & 3.083599 & 0.345244 & $\mathrm{O}$ & 1.904749 & -3.258328 & -0.727985 \\
\hline $\mathrm{O}$ & 1.875223 & 5.281509 & -0.505776 & $\mathrm{O}$ & -2.494117 & -4.633492 & -1.927853 \\
\hline $\mathrm{O}$ & 3.914351 & 3.791446 & -1.086063 & $\mathrm{O}$ & -4.355930 & -2.82 & 38696 \\
\hline $\mathrm{O}$ & 4.367971 & -5.027597 & -1.703179 & $\mathrm{O}$ & -1.239733 & 4.96 & -0.779004 \\
\hline $\mathrm{O}$ & 2.309796 & -4.4 & -0.100432 & $\mathrm{O}$ & -3.672325 & 5.81 & -0.085878 \\
\hline $\mathrm{H}$ & -0.624934 & -0.332533 & -0.468159 & $\mathrm{H}$ & 0.618694 & 0.372845 & -0.224735 \\
\hline $\mathrm{H}$ & -6.544056 & -1.273648 & -2.209400 & $\mathrm{H}$ & 6.467834 & 1.672 & -1.756571 \\
\hline $\mathrm{H}$ & -4.649045 & -2.501493 & 1.485742 & $\mathrm{H}$ & 4.695143 & 1.58 & 2.193790 \\
\hline $\mathrm{H}$ & -2.412165 & 0.993910 & 1.610534 & $\mathrm{H}$ & 2.379447 & -1.706605 & 1.120087 \\
\hline $\mathrm{H}$ & -0.622905 & 1.9 & 2.983213 & $\mathrm{H}$ & 1.044616 & -3.0 & 367 \\
\hline $\mathrm{H}$ & 1.217635 & 1.5 & 4.585441 & $\mathrm{H}$ & -0.701982 & -3.3 & 4.228092 \\
\hline $\mathrm{H}$ & 1.35 & -2.5 & 3.4 & $\mathrm{H}$ & -1.635 & 0.8 & 3.956091 \\
\hline $\mathrm{H}$ & -0.507035 & -2.18 & 1.869339 & $\mathrm{H}$ & 0.118903 & 1.1 & 2.219334 \\
\hline $\mathrm{H}$ & -0.645586 & 5.1 & 0.091805 & $\mathrm{H}$ & 0.039346 & -4.9 & 573 \\
\hline $\mathrm{H}$ & 4.719074 & 755 & -1.399607 & $\mathrm{H}$ & -4.834764 & -0.26 & -1.495371 \\
\hline $\mathrm{H}$ & 4.843 & -0.6 & -2.616097 & $\mathrm{H}$ & -1.026693 & 2.2 & 656 \\
\hline $\mathrm{H}$ & 5.516349 & -2.99 & -2.870159 & $\mathrm{H}$ & -5.504203 & 3.98 & 0.173620 \\
\hline $\mathrm{H}$ & 1.714 & -1.8 & 0.0 & $\mathrm{H}$ & -5.10 & 1.5 & 11 \\
\hline $\mathrm{H}$ & -3.812213 & 0.8 & -2.914199 & $\mathrm{H}$ & 3.664574 & -0.0 & -3.0 \\
\hline $\mathrm{H}$ & -6.64 & -3.2 & 1000 & $\mathrm{H}$ & 6.6 & 2.5 & 323 \\
\hline $\mathrm{H}$ & 2.67 & 0.0 & 5.4 & $\mathrm{H}$ & -2.328663 & -2.26 & 914 \\
\hline $\mathrm{H}$ & -2.566528 & 4.0 & 0.33 & $\mathrm{H}$ & 2.088030 & -4.1 & 1960 \\
\hline $\mathrm{H}$ & 2.807281 & 669 & -0.748199 & $\mathrm{H}$ & -3.393371 & -4.1 & -2.004396 \\
\hline $\mathrm{H}$ & 3.764048 & -5.5 & -1.166472 & $\mathrm{H}$ & -0.407274 & 4.5 & -1.0 \\
\hline $\mathrm{H}$ & 1.556422 & -4.1 & 071 & $\mathrm{H}$ & 471 & 27 & -0.2 \\
\hline \multicolumn{4}{|c|}{$2 \mathrm{~b} 1$} & \multicolumn{4}{|c|}{$2 \mathrm{~b} 2$} \\
\hline $\mathrm{C}$ & -1.21 & -1.5 & 879 & $\mathrm{C}$ & 0.129728 & -1.85 & -0.375539 \\
\hline $\mathrm{C}$ & -2.371550 & -1.304334 & -0.949080 & $\mathrm{C}$ & -0.953372 & -2.072059 & -1.434544 \\
\hline $\mathrm{C}$ & -3.334341 & -0.304985 & -0.581675 & $\mathrm{C}$ & -2.327181 & -1.90 & -1.055861 \\
\hline $\mathrm{C}$ & -4.444464 & 0.012822 & -1.429310 & $\mathrm{C}$ & -3.390199 & -2.070566 & -2.000170 \\
\hline $\mathrm{C}$ & -5.418853 & 0.911851 & -1.007682 & $\mathrm{C}$ & -4.717707 & -1.98 & -1.590847 \\
\hline $\mathrm{C}$ & -5.328872 & 1.460333 & 0.274900 & $\mathrm{C}$ & -5.000502 & -1.781644 & -0.238006 \\
\hline $\mathrm{C}$ & -4.274681 & & 8560 & $\mathrm{C}$ & -3.983430 & -1.6 & 0.723481 \\
\hline $\mathrm{C}$ & -3.289295 & 0.270076 & 0.715944 & $\mathrm{C}$ & -2.661286 & -1.708862 & 0.308749 \\
\hline $\mathrm{O}$ & -2.313168 & -0.064385 & 1.592931 & $\mathrm{O}$ & -1.701619 & -1.644188 & 1.265101 \\
\hline $\mathrm{C}$ & -1.020956 & -0.428016 & 1.018324 & $\mathrm{C}$ & -0.402106 & -1.117147 & 0.873589 \\
\hline $\mathrm{C}$ & -0.106473 & -0.743200 & 2.177173 & $\mathrm{C}$ & 0.497559 & -1.254387 & 2.080486 \\
\hline $\mathrm{C}$ & 0.940835 & 0.126994 & 2.506464 & $\mathrm{C}$ & 1.230693 & -0.166848 & 2.570516 \\
\hline $\mathrm{C}$ & 1.781596 & -0.127279 & 3.585461 & $\mathrm{C}$ & 2.070654 & -0.297746 & 3.672654 \\
\hline $\mathrm{C}$ & 1.576749 & -1.267864 & 4.367967 & $\mathrm{C}$ & 2.183943 & -1.535048 & 4.313605 \\
\hline $\mathrm{C}$ & 0.525720 & -2.140847 & 4.062651 & $\mathrm{C}$ & 1.450149 & -2.630764 & 3.842877 \\
\hline $\mathrm{C}$ & -0.304230 & -1.874980 & 2.977246 & $\mathrm{C}$ & 0.619343 & -2.486205 & 2.735533 \\
\hline $\mathrm{C}$ & 0.037198 & -2.105880 & -0.690135 & $\mathrm{C}$ & 1.424964 & -1.305079 & -0.972211 \\
\hline
\end{tabular}




\begin{tabular}{|c|c|c|c|c|c|c|c|}
\hline $\mathrm{C}$ & 0.074292 & -3.475798 & -1.049184 & $\mathrm{C}$ & 2.314238 & -2.241187 & -1.556790 \\
\hline $\mathrm{C}$ & 1.236032 & -4.117944 & -1.475262 & $\mathrm{C}$ & 3.611913 & -1.919057 & -1.944972 \\
\hline $\mathrm{C}$ & 2.407888 & -3.385926 & -1.643122 & $\mathrm{C}$ & 4.048160 & -0.600573 & -1.856479 \\
\hline $\mathrm{C}$ & 2.388536 & -1.978386 & -1.430773 & $\mathrm{C}$ & 3.141432 & 0.408042 & -1.422169 \\
\hline $\mathrm{C}$ & 3.571301 & -1.169236 & -1.689776 & $\mathrm{C}$ & 3.536435 & 1.809758 & -1.437967 \\
\hline $\mathrm{C}$ & 3.403834 & 0.256874 & -1.503260 & $\mathrm{C}$ & 2.508980 & 2.751245 & -1.051736 \\
\hline $\mathrm{C}$ & 2.223664 & 0.780087 & -1.084383 & $\mathrm{C}$ & 1.267424 & 2.337129 & -0.693734 \\
\hline $\mathrm{O}$ & 1.145003 & -0.010859 & -0.818530 & $\mathrm{O}$ & 0.944290 & 1.008296 & -0.629834 \\
\hline $\mathrm{C}$ & 1.195441 & -1.377886 & -0.982156 & $\mathrm{C}$ & 1.848731 & 0.030532 & -1.003479 \\
\hline $\mathrm{C}$ & 1.933577 & 2.208122 & -0.888447 & $\mathrm{C}$ & 0.150022 & 0176 & -0.348119 \\
\hline $\mathrm{C}$ & 2.973046 & 3.104377 & -0.569011 & $\mathrm{C}$ & 0.068004 & 4.506667 & -0.926954 \\
\hline $\mathrm{C}$ & 2.708232 & 4.455793 & -0.419581 & $\mathrm{C}$ & -0.969123 & 5.376659 & -0.600284 \\
\hline $\mathrm{C}$ & 1.399071 & 4.953032 & -0.577992 & $\mathrm{C}$ & -1.950193 & 4.989221 & 0.309724 \\
\hline $\mathrm{C}$ & 0.368332 & 4.069703 & -0.888663 & $\mathrm{C}$ & -1.879623 & 3.708512 & 0.889922 \\
\hline $\mathrm{C}$ & 0.631499 & 2.709652 & -1.041116 & $\mathrm{C}$ & -0.848328 & 2.841508 & 0.565738 \\
\hline $\mathrm{O}$ & -2.487780 & -2.009096 & -1.978230 & $\mathrm{O}$ & -0.609019 & -2.469949 & -2.570500 \\
\hline $\mathrm{O}$ & -4.565857 & -0.546777 & -2.6 & $\mathrm{O}$ & -3.130258 & -2.3 & -3.289777 \\
\hline $\mathrm{O}$ & -6.312815 & 2.319486 & 0.639435 & $\mathrm{O}$ & -6.310371 & -1.72 & 0.108372 \\
\hline $\mathrm{O}$ & 2.428303 & -1.475010 & 575 & $\mathrm{O}$ & 3.0 & -1.6 & 5.392251 \\
\hline $\mathrm{O}$ & -1.060244 & -4.226556 & -0.967230 & $\mathrm{O}$ & 1.905145 & -3.527631 & -1.753590 \\
\hline $\mathrm{O}$ & 3.522603 & -4.002541 & -2.052392 & $\mathrm{O}$ & 5.291757 & -0.28 & -2.236525 \\
\hline $\mathrm{O}$ & 4.655751 & -1.667279 & -2.068162 & $\mathrm{O}$ & 4.685783 & 2.180080 & -1.768067 \\
\hline $\mathrm{O}$ & 3.645968 & 5.407955 & -0.097967 & $\mathrm{O}$ & -2.889526 & 3.4 & 1.778408 \\
\hline $\mathrm{O}$ & 1.143935 & 6.276621 & -0.424932 & $\mathrm{O}$ & -2.962609 & 5.834867 & 0.626795 \\
\hline $\mathrm{H}$ & -1.585166 & -2.442452 & 0.603458 & $\mathrm{H}$ & 0.376769 & -2.8 & -0.044998 \\
\hline $\mathrm{H}$ & -3.847097 & -1.224098 & -2.723369 & $\mathrm{H}$ & -2.150036 & -2.434537 & -3.375295 \\
\hline $\mathrm{H}$ & -6.252051 & 1.158897 & -1.654115 & $\mathrm{H}$ & -5.520311 & -2.101280 & -2.308614 \\
\hline $\mathrm{H}$ & -6.155502 & 2.622470 & 1.547885 & $\mathrm{H}$ & -6.386030 & -1.605924 & 1.068766 \\
\hline $\mathrm{H}$ & -4.212429 & 1.568401 & 2.146243 & $\mathrm{H}$ & -4.208593 & -1.509047 & 1.776757 \\
\hline $\mathrm{H}$ & -0.657182 & 0.458915 & 0.498545 & $\mathrm{H}$ & -0.553763 & -0.067082 & 0.628214 \\
\hline $\mathrm{H}$ & 1.102533 & 1.019231 & 1.907673 & $\mathrm{H}$ & 1.155458 & 0.800374 & 2.082435 \\
\hline $\mathrm{H}$ & 2.595778 & 0.544489 & 3.837553 & $\mathrm{H}$ & 2.643008 & 0.544446 & 4.047715 \\
\hline $\mathrm{H}$ & 2.187437 & -2.301277 & 5.861962 & $\mathrm{H}$ & 3.018105 & -2.521468 & 5.729284 \\
\hline $\mathrm{H}$ & 0.357696 & -3.026004 & 4.673284 & $\mathrm{H}$ & 1.529149 & -3.594475 & 4.342761 \\
\hline $\mathrm{H}$ & -1.121397 & -2.558675 & 2.765185 & $\mathrm{H}$ & 0.044231 & -3.340433 & 2.389876 \\
\hline $\mathrm{H}$ & -1.781558 & -3.676341 & -1.343409 & $\mathrm{H}$ & 0.999839 & -3.473023 & -2.128986 \\
\hline $\mathrm{H}$ & 1.209317 & -5.173435 & -1.718327 & $\mathrm{H}$ & 4.254309 & -2.682591 & -2.366984 \\
\hline $\mathrm{H}$ & 4.218502 & -3.291342 & -2.151215 & $\mathrm{H}$ & 5.375855 & 0.703495 & -2.143197 \\
\hline $\mathrm{H}$ & 4.237977 & 0.901317 & -1.750644 & $\mathrm{H}$ & 2.760438 & 3.804097 & -1.046027 \\
\hline $\mathrm{H}$ & 3.983473 & 2.734832 & -0.413426 & $\mathrm{H}$ & -0.805742 & 1.868847 & 1.044339 \\
\hline $\mathrm{H}$ & 4.524071 & 5.003142 & -0.032629 & $\mathrm{H}$ & -2.790553 & 2.519180 & 2.113315 \\
\hline $\mathrm{H}$ & 1.986004 & 6.720207 & -0.221234 & $\mathrm{H}$ & -3.540871 & 5.380380 & 1.264136 \\
\hline $\mathrm{H}$ & -0.634019 & 4.464992 & -1.016611 & $\mathrm{H}$ & -1.040847 & 6.359348 & -1.054701 \\
\hline $\mathrm{H}$ & -0.175049 & 2.037034 & -1.309579 & $\mathrm{H}$ & 0.804377 & 4.812835 & -1.661940 \\
\hline \multicolumn{4}{|c|}{$2 \mathrm{b3}$} & \multicolumn{4}{|c|}{$2 b$} \\
\hline
\end{tabular}




\begin{tabular}{|c|c|c|c|c|c|c|c|}
\hline $\mathrm{C}$ & 0.155824 & -1.888393 & -0.187622 & $\mathrm{C}$ & -0.754228 & -1.760608 & -0.496895 \\
\hline $\mathrm{C}$ & -0.994807 & -2.193048 & -1.148643 & $\mathrm{C}$ & -1.841983 & -1.385730 & -1.505866 \\
\hline $\mathrm{C}$ & -2.340419 & -1.974511 & -0.691483 & $\mathrm{C}$ & -3.019865 & -0.722009 & 27877 \\
\hline $\mathrm{C}$ & -3.467022 & -2.196303 & -1.546223 & $\mathrm{C}$ & -4.069407 & -0.325259 & -1.917530 \\
\hline $\mathrm{C}$ & -4.762464 & -2.059082 & -1.055360 & $\mathrm{C}$ & -5.239048 & 0.241010 & -1.420272 \\
\hline $\mathrm{C}$ & -4.948815 & -1.765808 & 0.299061 & $\mathrm{C}$ & -5.397454 & 625 & 7785 \\
\hline $\mathrm{C}$ & -3.867523 & -1.579916 & 1.177343 & $\mathrm{C}$ & -4.404831 & -0.042297 & 7900 \\
\hline $\mathrm{C}$ & -2.576628 & -1.688261 & 230 & $\mathrm{C}$ & -3.229340 & 1903 & 536 \\
\hline $\mathrm{O}$ & -1.548927 & -1.565505 & 1.551578 & $\mathrm{O}$ & -2.317267 & -1.034240 & 1.263401 \\
\hline $\mathrm{C}$ & -0.306905 & -1.021415 & 218 & $\mathrm{C}$ & -0.921238 & 518 & 3097 \\
\hline $\mathrm{C}$ & 0.680105 & -0.945037 & 2.143993 & $\mathrm{C}$ & -0.139666 & -1.661224 & 9952 \\
\hline $\mathrm{C}$ & 1.105704 & 0.299481 & 2.626029 & $\mathrm{C}$ & -0.504717 & -2.922891 & 716 \\
\hline $\mathrm{C}$ & 2.004988 & 0.397646 & 3.682892 & $\mathrm{C}$ & 0.207743 & -3.527470 & 3.502009 \\
\hline $\mathrm{C}$ & 2.489722 & -0.766104 & 4.287983 & $\mathrm{C}$ & 1.315552 & -2.874611 & 4.0 \\
\hline $\mathrm{C}$ & 2.06 & -2.0 & 767 & $\mathrm{C}$ & 059 & 246 & 765 \\
\hline $\mathrm{C}$ & 1.168317 & -2.10 & 256 & $\mathrm{C}$ & 0.958974 & 289 & 269 \\
\hline $\mathrm{C}$ & 1.41 & -1.4 & -0.9 & $\mathrm{C}$ & 514 & -1. & 571 \\
\hline $\mathrm{C}$ & 2.274200 & -2.396756 & -1.465680 & $\mathrm{C}$ & 1.060863 & -2.861481 & 5169 \\
\hline $\mathrm{C}$ & 3.550589 & -2.101373 & 2328 & $\mathrm{C}$ & 2.374872 & -3.06 & 203 \\
\hline $\mathrm{C}$ & 3.988960 & -0.779501 & -1.969467 & $\mathrm{C}$ & 3.323182 & -2.067213 & 833 \\
\hline $\mathrm{C}$ & 3.102496 & 0.25 & 19 & $\mathrm{C}$ & 2.922005 & -0.8 & 296 \\
\hline $\mathrm{C}$ & 3.485224 & 1.660378 & -1.687229 & $\mathrm{C}$ & 3.865244 & 0.265975 & 477 \\
\hline $\mathrm{C}$ & 2.462004 & 2.62 & 375 & $\mathrm{C}$ & & 45 & 86 \\
\hline $\mathrm{C}$ & 1.246200 & 2.220996 & 7056 & $\mathrm{C}$ & 2.019843 & 055 & 274 \\
\hline $\mathrm{O}$ & 0.9 & 0.8 & -0 & $\mathrm{O}$ & 14 & 0.5 & -0 . \\
\hline $\mathrm{C}$ & $1.8 ?$ & -0.0 & $-1 .($ & $\mathrm{C}$ & 415 & -0.691676 & $-1 .($ \\
\hline $\mathrm{C}$ & 0.118124 & 3.090397 & -0.514814 & $\mathrm{C}$ & 642 & 2.82 & 903 \\
\hline $\mathrm{C}$ & -1.203473 & 2.607601 & -0.606430 & $\mathrm{C}$ & 171 & 292 & 534 \\
\hline $\mathrm{C}$ & -2.271638 & 3.428597 & 3605 & $\mathrm{C}$ & 1.290514 & 5.246729 & 611 \\
\hline $\mathrm{C}$ & -2.054110 & 4.754869 & 7718 & $\mathrm{C}$ & 987 & 000 & 537 \\
\hline $\mathrm{C}$ & -0.749019 & 5.234466 & 0.232443 & $\mathrm{C}$ & -0.258638 & 365 & 570 \\
\hline $\mathrm{C}$ & 0.327160 & 4.410592 & -0.088383 & $\mathrm{C}$ & 0.308405 & 2.796752 & 883 \\
\hline $\mathrm{O}$ & -0.732257 & -2.684423 & -2.268972 & $\mathrm{O}$ & -1.7 & -1.74 & -2.6 \\
\hline $\mathrm{O}$ & -3.296812 & -2.531911 & -2.831034 & $\mathrm{O}$ & -3.945961 & -0.490157 & -3.2 \\
\hline $\mathrm{O}$ & -6.231300 & -1.661107 & 0.727326 & $\mathrm{O}$ & -6.562433 & 0.913648 & 0.3 \\
\hline $\mathrm{O}$ & 3.368605 & -0.619312 & 5.321361 & $\mathrm{O}$ & 1.980964 & -3.515023 & 163 \\
\hline $\mathrm{O}$ & 1.860989 & -3.695244 & -1.535045 & $\mathrm{O}$ & 0.152150 & -3.824070 & -2.167787 \\
\hline $\mathrm{O}$ & 5.213502 & -0.495420 & -2.426708 & $\mathrm{O}$ & 4.583830 & -2.255780 & -2.451843 \\
\hline $\mathrm{O}$ & 4.614173 & 2.011191 & -2.097494 & $\mathrm{O}$ & 5.065739 & 0.165962 & -1.676902 \\
\hline $\mathrm{O}$ & -3.101920 & 5.556846 & 0.453137 & $\mathrm{O}$ & 1.678347 & 6.510214 & -0.283301 \\
\hline $\mathrm{O}$ & -3.592724 & 3.055097 & -0.345407 & $\mathrm{O}$ & -0.330919 & 6.362005 & 3902 \\
\hline $\mathrm{H}$ & 0.412836 & -2.875458 & 0.228106 & $\mathrm{H}$ & -0.948746 & -2.825123 & -0.290460 \\
\hline $\mathrm{H}$ & -2.326871 & -2.684893 & -2.968683 & $\mathrm{H}$ & -3.102509 & -0.986579 & -3.396809 \\
\hline $\mathrm{H}$ & -5.614114 & -2.219332 & -1.705283 & $\mathrm{H}$ & -6.027513 & 0.550597 & -2.095284 \\
\hline $\mathrm{H}$ & -6.240117 & -1.490616 & 1.682768 & $\mathrm{H}$ & -6.567034 & 0.937921 & 1.363625 \\
\hline $\mathrm{H}$ & -4.017032 & -1.361745 & 2.230389 & $\mathrm{H}$ & -4.537977 & 0.048065 & 1.941650 \\
\hline $\mathrm{H}$ & -0.543734 & -0.014069 & 0.664691 & $\mathrm{H}$ & -0.636980 & 0.017752 & 0.733005 \\
\hline $\mathrm{H}$ & 0.729360 & 1.209103 & 2.165858 & $\mathrm{H}$ & -1.370052 & -3.432944 & 2.060699 \\
\hline
\end{tabular}




\begin{tabular}{|c|c|c|c|c|c|c|c|}
\hline $\mathrm{H}$ & 2.339495 & 1.361773 & 4.051838 & $\mathrm{H}$ & -0.075119 & -4.501844 & 3.887150 \\
\hline $\mathrm{H}$ & 3.636636 & -1.495891 & 5.638583 & $\mathrm{H}$ & 2.737337 & -2.973987 & 5.338910 \\
\hline $\mathrm{H}$ & 2.432823 & -2.926503 & 4.301941 & $\mathrm{H}$ & 2.544616 & -1.099173 & 4.013389 \\
\hline $\mathrm{H}$ & 0.840854 & -3.081186 & 2.432348 & $\mathrm{H}$ & 1.260222 & -0.035905 & 2.200405 \\
\hline $\mathrm{H}$ & 0.931824 & -3.672327 & -1.848116 & $\mathrm{H}$ & -0.653348 & -3.351314 & -2.471603 \\
\hline $\mathrm{H}$ & 4.176171 & -2.893969 & -2.335217 & $\mathrm{H}$ & 2.637799 & 223 & 2238 \\
\hline $\mathrm{H}$ & 5.298199 & 0.500873 & -2.406671 & $\mathrm{H}$ & 5.075739 & -1.408397 & 041 \\
\hline $\mathrm{H}$ & 2.679954 & 3.672302 & $-1.4^{\prime}$ & $\mathrm{H}$ & 0993 & 022 & 323 \\
\hline $\mathrm{H}$ & -1.389006 & 1.595711 & -0.954483 & $\mathrm{H}$ & 2.655333 & 4.121979 & 1482 \\
\hline $\mathrm{H}$ & -3.666387 & 2.131044 & -0.629700 & $\mathrm{H}$ & 2.406896 & 865 & 9419 \\
\hline $\mathrm{H}$ & -3.918435 & 5.044089 & 0.319564 & $\mathrm{H}$ & 0.136889 & 515 & 898 \\
\hline $\mathrm{H}$ & -0.594118 & 6.253798 & 0.57 & $\mathrm{H}$ & -1.079392 & 328 & 065 \\
\hline $\mathrm{H}$ & 1.337262 & 4.79 & 0.0 & $\mathrm{H}$ & -0.0 & 1.8 & 681 \\
\hline \multicolumn{4}{|c|}{$2 \mathrm{b5}$} & \multicolumn{4}{|c|}{$2 \mathrm{~b} 7$} \\
\hline $\mathrm{C}$ & -1.102040 & 1.08 & -0.9 & $\mathrm{C}$ & 0.9 & -0.9 & $-1 .($ \\
\hline $\mathrm{C}$ & 0.254014 & 1.25 & -1.6 & $\mathrm{C}$ & -0.5 & -0.9 & -1 . \\
\hline $\mathrm{C}$ & 1.116226 & 2.295319 & -1.177342 & $\mathrm{C}$ & -1.385826 & -1.93 & -0.9 \\
\hline $\mathrm{C}$ & 2.355322 & 2.608213 & -1.8 & $\mathrm{C}$ & 2453 & -2.0 & 165 \\
\hline $\mathrm{C}$ & 3.130058 & 3.680047 & -1.383307 & $\mathrm{C}$ & -3.5 & -3.070619 & -0.8 \\
\hline $\mathrm{C}$ & 2.676897 & 4.454063 & -0.3 & $\mathrm{C}$ & -3.0 & -4.0 & $-0 .($ \\
\hline $\mathrm{C}$ & 1.473115 & 4.170206 & 0.3 & $\mathrm{C}$ & -1.669999 & -3.9 & 300 \\
\hline $\mathrm{C}$ & 0.710142 & 3.09 & -0.0 & $\mathrm{C}$ & -0.8 & -2.8 & -0. \\
\hline $\mathrm{O}$ & -0.448211 & 2.84 & 0.5 & $\mathrm{O}$ & 65 & -2.8 & 298 \\
\hline $\mathrm{C}$ & -0.969772 & 1.49 & 0.4 & $\mathrm{C}$ & 1.0 & -1.5 & 303 \\
\hline $\mathrm{C}$ & -2.263609 & 1.46 & 1.2 & $\mathrm{C}$ & 964 & -1.7 & 792 \\
\hline $\mathrm{C}$ & -2.490718 & 0.481319 & 2.236735 & $\mathrm{C}$ & 2.920285 & -0.8 & 6642 \\
\hline $\mathrm{C}$ & -3.684272 & 0.427597 & 2.950599 & $\mathrm{C}$ & 4.213479 & -1.0 & 246 \\
\hline $\mathrm{C}$ & -4.675049 & 1.384311 & 2.710835 & $\mathrm{C}$ & 468 & -2.0 & 323 \\
\hline $\mathrm{C}$ & -4.460340 & 2.383675 & 1.75 & $\mathrm{C}$ & 310 & -2.8 & 682 \\
\hline $\mathrm{C}$ & -3.265444 & 2.417804 & 1.039926 & $\mathrm{C}$ & 3.280826 & -2.70 & 125 \\
\hline $\mathrm{C}$ & -1.730097 & -0.271341 & -1.197098 & $\mathrm{C}$ & 1.630922 & 95 & -1 . \\
\hline $\mathrm{C}$ & -3.034161 & -0.395197 & -1.711450 & $\mathrm{C}$ & 2.900912 & 0.5 & -1.7 \\
\hline $\mathrm{C}$ & -3.665646 & -1.630426 & -1.886268 & $\mathrm{C}$ & 3.631227 & 1.72 & 9096 \\
\hline $\mathrm{C}$ & -3.000864 & -2.805673 & -1.547047 & $\mathrm{C}$ & 3.110331 & 391 & 2134 \\
\hline $\mathrm{C}$ & -1.678876 & -2.735748 & -1.028531 & $\mathrm{C}$ & 1.822254 & 2.803447 & 3360 \\
\hline $\mathrm{C}$ & -0.950404 & -3.942340 & -0.662092 & $\mathrm{C}$ & 1.238041 & 3262 & 0.103212 \\
\hline $\mathrm{C}$ & 0.394859 & -3.726619 & -0.175471 & $\mathrm{C}$ & -0.107926 & 3.796718 & 0.602086 \\
\hline $\mathrm{C}$ & 0.913046 & -2.475775 & -0.052533 & $\mathrm{C}$ & -0.751654 & 2.604247 & 4278 \\
\hline $\mathrm{O}$ & 0.188078 & -1.365663 & -0.374805 & $\mathrm{O}$ & -0.132695 & 1.503577 & -0.035023 \\
\hline $\mathrm{C}$ & -1.085717 & -1.470446 & -0.879686 & $\mathrm{C}$ & 1.119389 & 488 & -0.589124 \\
\hline $\mathrm{C}$ & 2.267304 & -2.146935 & 0.410689 & $\mathrm{C}$ & -2.150574 & 2.336155 & 0.843777 \\
\hline $\mathrm{C}$ & 2.907463 & -2.970436 & 1.357588 & $\mathrm{C}$ & -2.789893 & 2.993028 & 1.904408 \\
\hline $\mathrm{C}$ & 4.194844 & -2.677491 & 1.776273 & $\mathrm{C}$ & -4.136485 & 2.748930 & 2.172317 \\
\hline $\mathrm{C}$ & 4.876668 & -1.554850 & 1.266196 & $\mathrm{C}$ & -4.860002 & 1.855374 & 1.383670 \\
\hline $\mathrm{C}$ & 4.245510 & -0.740145 & 0.328942 & $\mathrm{C}$ & -4.223384 & 1.194628 & 0.312108 \\
\hline $\mathrm{C}$ & 2.949985 & -1.029466 & -0.093664 & $\mathrm{C}$ & -2.883481 & 1.430698 & 0.052748 \\
\hline $\mathrm{O}$ & 0.563762 & 0.547803 & -2.666772 & $\mathrm{O}$ & -0.913195 & -0.137692 & -2.425685 \\
\hline $\mathrm{O}$ & 2.793663 & 1.871878 & -2.850277 & $\mathrm{O}$ & -3.296429 & -1.119705 & -2.195070 \\
\hline
\end{tabular}




\begin{tabular}{|l|r|r|r||r|r|r|r|}
\hline $\mathrm{O}$ & 3.467360 & 5.490186 & 0.071602 & $\mathrm{O}$ & -3.829638 & -5.003760 & 0.409699 \\
$\mathrm{O}$ & -5.826784 & 1.296732 & 3.441259 & $\mathrm{O}$ & 6.303171 & -2.147556 & 2.380398 \\
$\mathrm{O}$ & -3.675542 & 0.762740 & -2.039625 & $\mathrm{O}$ & 3.405324 & -0.586370 & -2.349570 \\
$\mathrm{O}$ & -3.611106 & -3.984667 & -1.710406 & $\mathrm{O}$ & 3.822702 & 3.993273 & -1.123339 \\
$\mathrm{O}$ & -1.451192 & -5.084538 & -0.778277 & $\mathrm{O}$ & 1.852592 & 5.062088 & 0.180094 \\
$\mathrm{O}$ & 4.902887 & -3.405215 & 2.705103 & $\mathrm{O}$ & -5.022526 & 0.355135 & -0.412481 \\
$\mathrm{O}$ & 6.135618 & -1.269118 & 1.685268 & $\mathrm{O}$ & -6.171694 & 1.617865 & 1.639602 \\
$\mathrm{H}$ & -1.762674 & 1.829567 & -1.465705 & $\mathrm{H}$ & 1.466688 & -1.611084 & -1.758829 \\
$\mathrm{H}$ & 4.065748 & 3.915305 & -1.875781 & $\mathrm{H}$ & -4.596057 & -3.144769 & -1.192370 \\
$\mathrm{H}$ & 1.138110 & 4.754626 & 1.203397 & $\mathrm{H}$ & -1.248041 & -4.653949 & 1.100893 \\
$\mathrm{H}$ & -0.240609 & 0.830017 & 0.973397 & $\mathrm{H}$ & 0.465462 & -0.890183 & 1.028323 \\
$\mathrm{H}$ & -1.720962 & -0.260097 & 2.436958 & $\mathrm{H}$ & 2.276597 & -0.132000 & 2.322413 \\
$\mathrm{H}$ & -3.860924 & -0.338586 & 3.698559 & $\mathrm{H}$ & 4.589347 & -0.410080 & 3.185396 \\
$\mathrm{H}$ & -5.227211 & 3.134469 & 1.570390 & $\mathrm{H}$ & 5.216067 & -3.644689 & 0.427290 \\
$\mathrm{H}$ & -3.106005 & 3.203001 & 0.307131 & $\mathrm{H}$ & 2.922049 & -3.357652 & -0.440452 \\
$\mathrm{H}$ & -4.675020 & -1.693178 & -2.283344 & $\mathrm{H}$ & 4.611458 & 1.784977 & -2.224037 \\
$\mathrm{H}$ & 1.001988 & -4.595218 & 0.046951 & $\mathrm{H}$ & -0.627679 & 4.661654 & 0.995119 \\
$\mathrm{H}$ & 2.382386 & -3.822290 & 1.782948 & $\mathrm{H}$ & -2.232221 & 3.676577 & 2.536948 \\
$\mathrm{H}$ & 4.783756 & 0.115135 & -0.066113 & $\mathrm{H}$ & -4.642497 & 3.240025 & 2.997261 \\
$\mathrm{H}$ & 2.484155 & -0.409372 & -0.849599 & $\mathrm{H}$ & -2.404758 & 0.949239 & -0.789084 \\
$\mathrm{H}$ & 2.079439 & 1.207932 & -3.051547 & $\mathrm{H}$ & -2.529609 & -0.569498 & -2.532760 \\
$\mathrm{H}$ & 3.050326 & 5.950197 & 0.817198 & $\mathrm{H}$ & -3.342825 & -5.596166 & 1.004630 \\
$\mathrm{H}$ & -6.420225 & 2.019304 & 3.183734 & $\mathrm{H}$ & 6.766083 & -2.875961 & 1.937902 \\
$\mathrm{H}$ & -4.559078 & 0.548604 & -2.377381 & $\mathrm{H}$ & 4.288621 & -0.389345 & -2.698133 \\
$\mathrm{H}$ & -2.958050 & -4.681087 & -1.406587 & $\mathrm{H}$ & 3.263858 & 4.665757 & -0.631959 \\
$\mathrm{H}$ & 4.388195 & -4.177470 & 2.983741 & $\mathrm{H}$ & -4.504377 & -0.090936 & -1.115881 \\
$\mathrm{H}$ & 6.397727 & -1.954631 & 2.324378 & $\mathrm{H}$ & -6.478227 & 0.972218 & 0.977291 \\
\hline
\end{tabular}

TABEL S3. Frequencies of (2R)-1 at B3LYP/6-31G* Level in the Gas Phase $\left(\mathrm{cm}^{-1}\right)$

\begin{tabular}{|c|c|}
\hline Species & Frequencies \\
\hline$(2 R)-1$ & $\begin{array}{l}\text { 22. 47. 68. 97. 129. 181. 224. 233. 241. 271. 295. 322. 362. 407. 407. 415. 458. 492. } 503 . \\
\text { 534. 572. 596. 624. 632. 634. 651. 661. 700. 712. 723. 737. 780. 788. 826. 843. 862. } 873 . \\
\text { 906. 930. 970. 998. 1000.1018. 1037. 1058. 1078. 1083. 1115. 1124. 1191. 1194. 1202. } \\
\text { 1212. 1218. 1242. 1278. 1288. 1317. 1343. 1364. 1366. 1389. 1409. 1420. 1474. 1479. } 1502 . \\
\text { 1514. 1547. 1549. 1644. 1647. 1667. 1690. 1714. 3021. 3052. 3128. 3176. 3187. 3197. 3205. } \\
\text { 3209. 3216. 3244. 3271. 3747. }\end{array}$ \\
\hline
\end{tabular}


TABLE S4. Frequencies of Conformers of $(2 R, 3 S)-2$ in the Gas Phase $\left(\mathrm{cm}^{-1}\right)$

\begin{tabular}{|c|c|}
\hline Species & Frequencies \\
\hline $2 a 1$ & 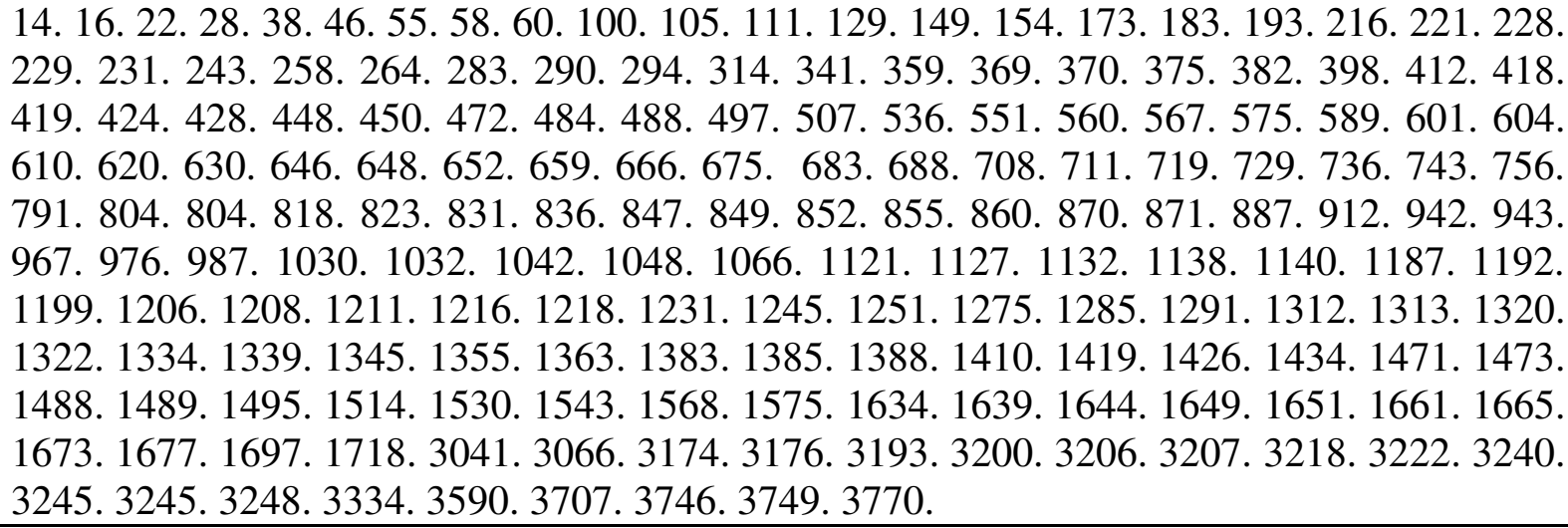 \\
\hline $2 a 2$ & 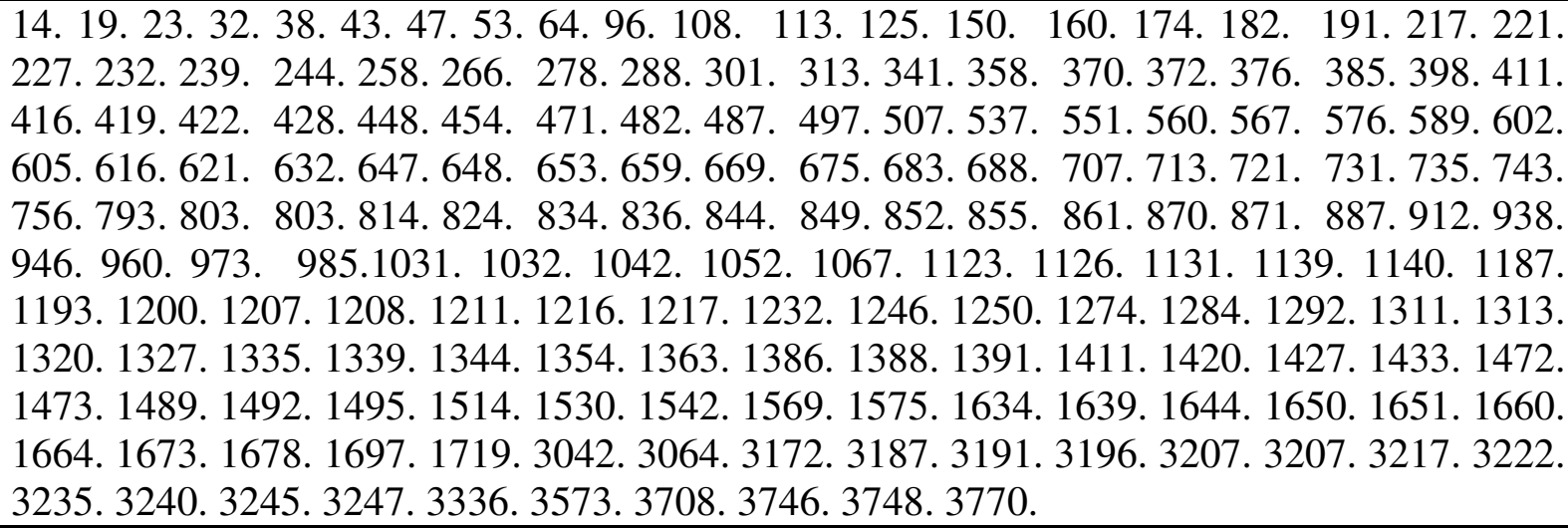 \\
\hline $2 a 3$ & 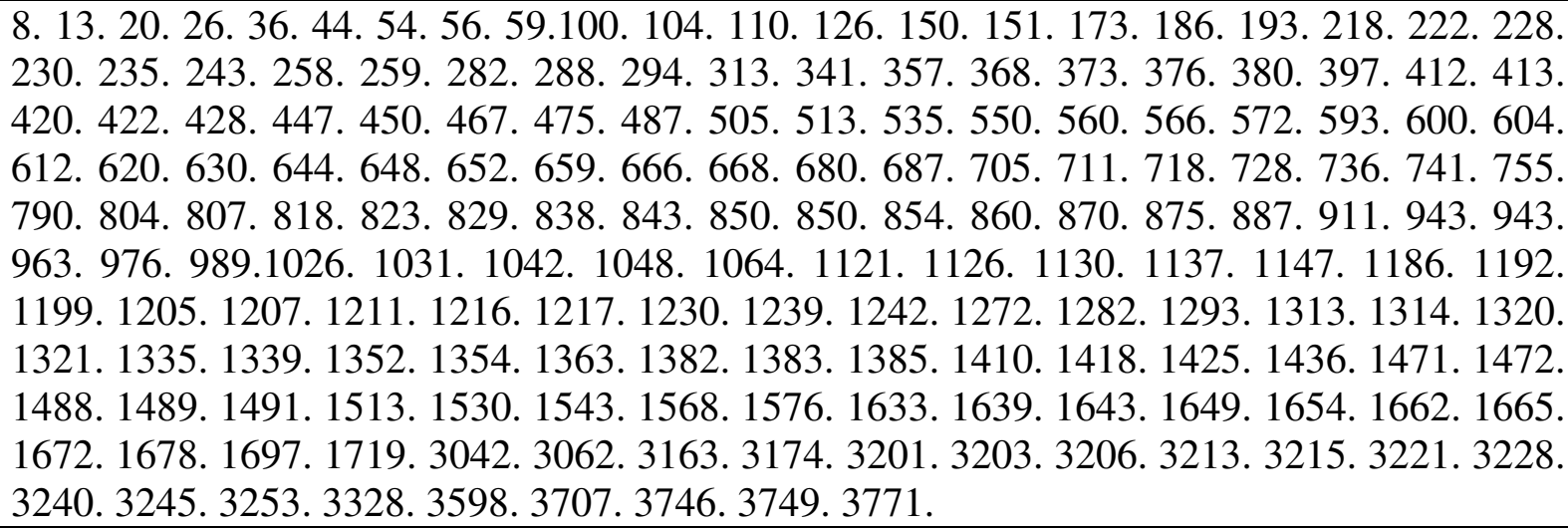 \\
\hline $2 a 4$ & 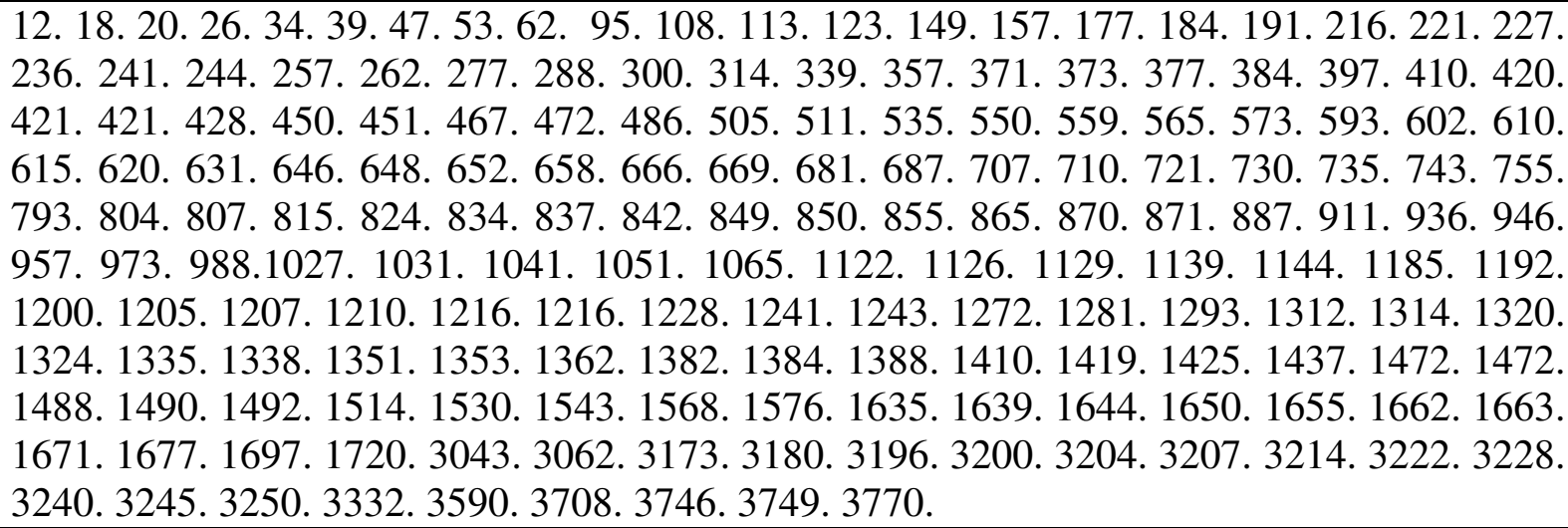 \\
\hline
\end{tabular}




\begin{tabular}{|c|c|}
\hline $2 \mathrm{a} 5$ & 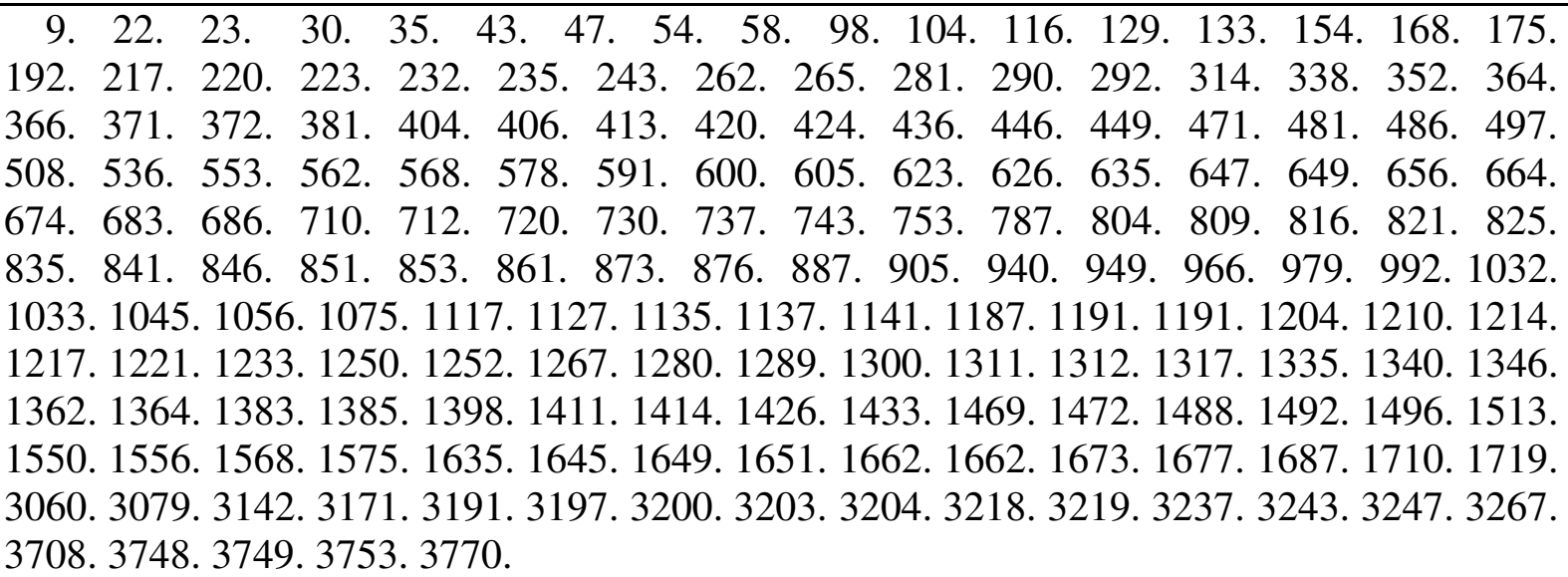 \\
\hline $2 a 6$ & 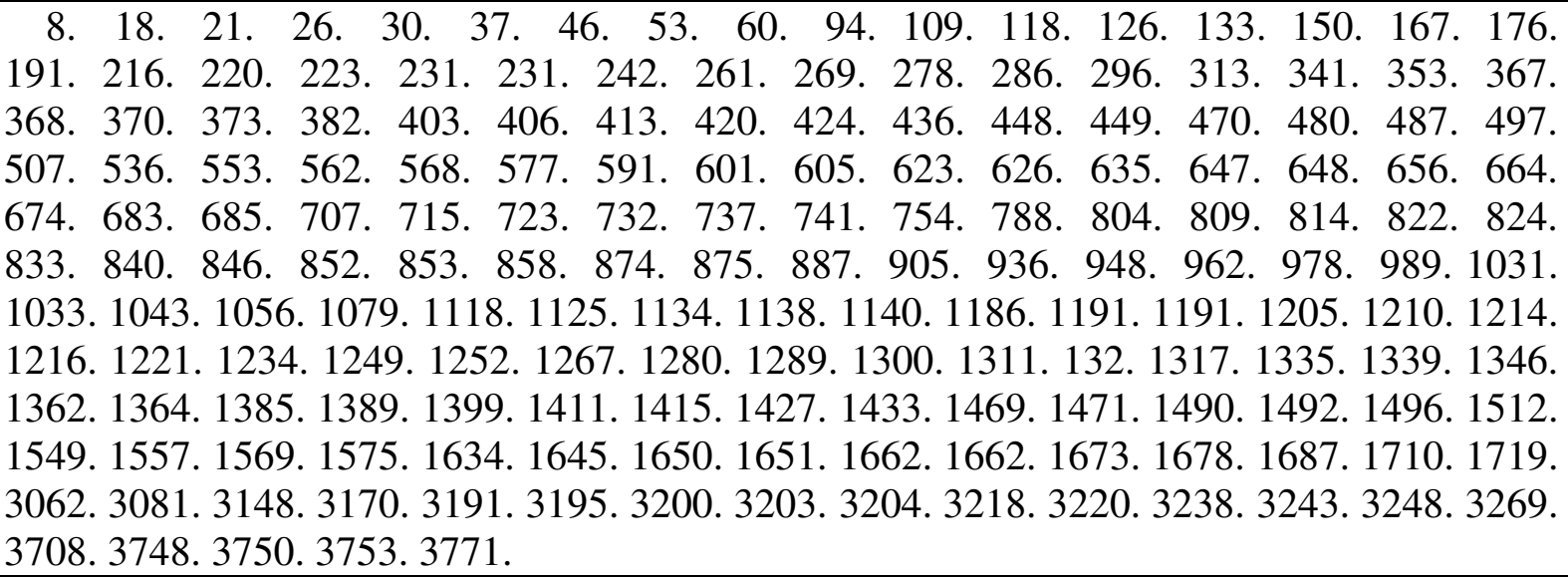 \\
\hline $2 \mathrm{a} 7$ & 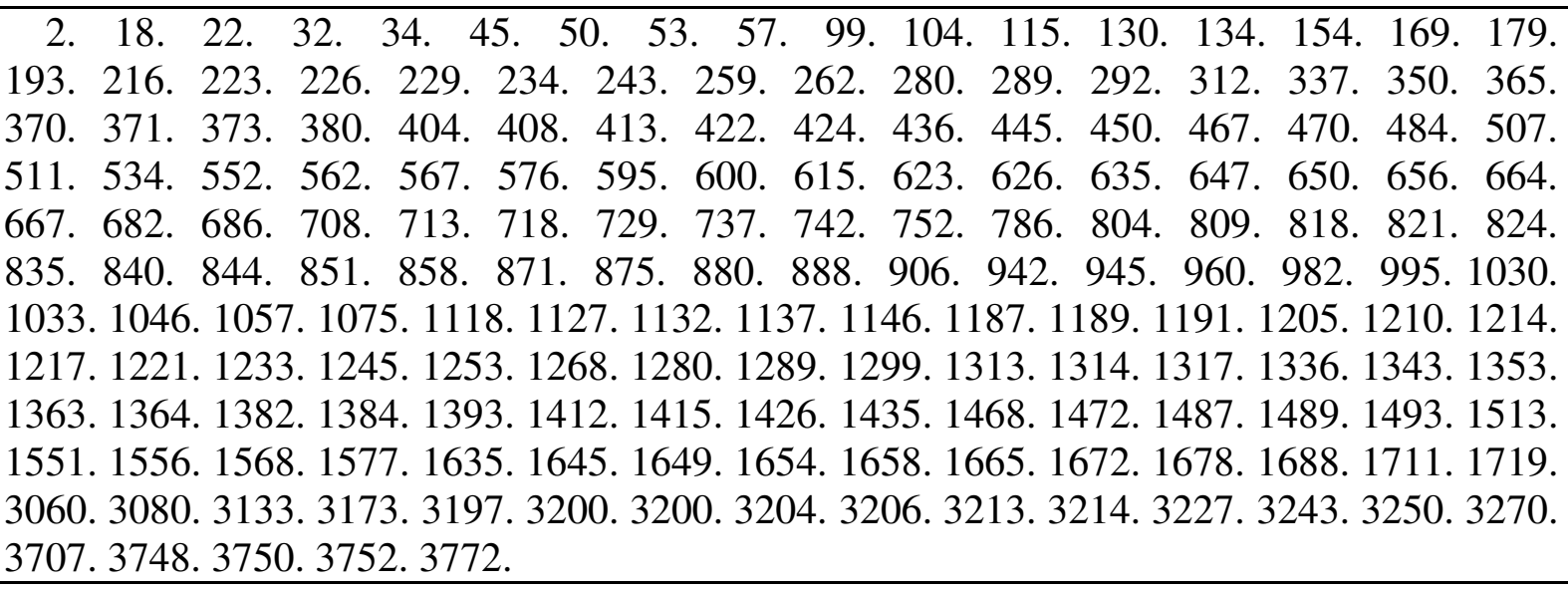 \\
\hline $2 \mathrm{a} 8$ & 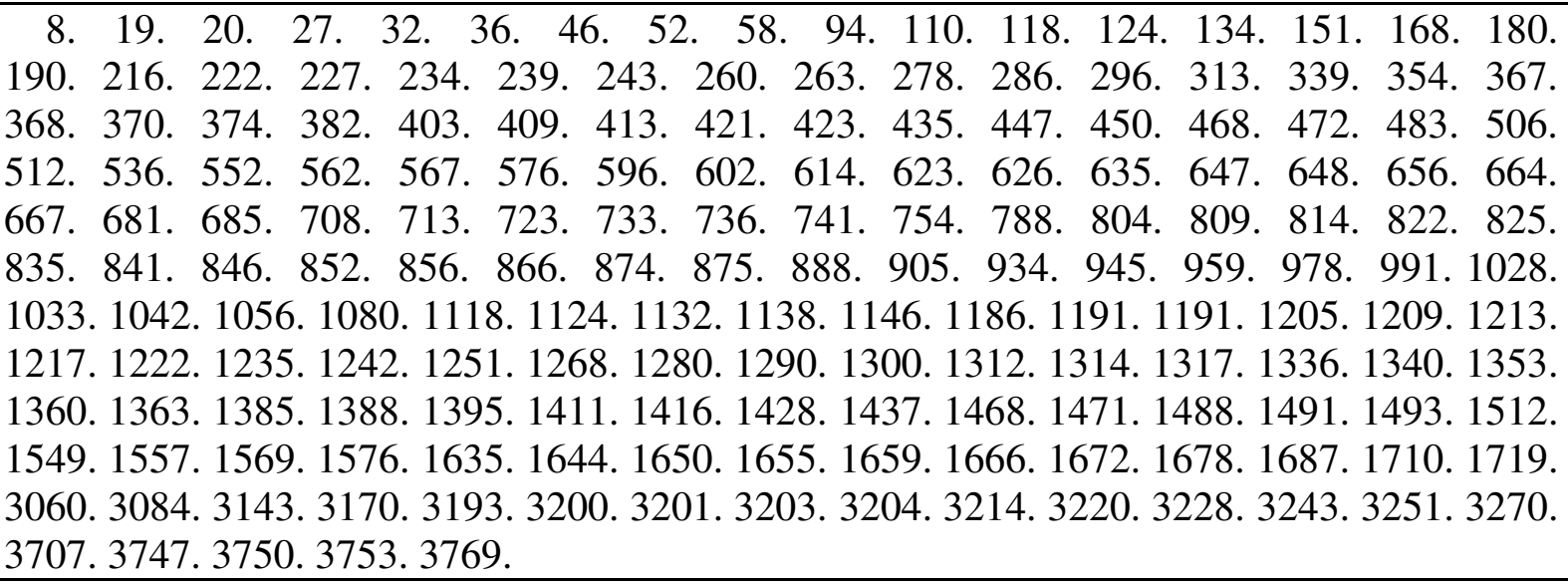 \\
\hline
\end{tabular}




\begin{tabular}{|c|c|}
\hline $2 \mathrm{~b} 1$ & $\begin{array}{l}\text { 9. 21. 24. 31. 38. 45. 51. 57. 67.107. 108. 119. 123. 150. 158. 166. 176. 191. 219. 220. } 227 . \\
\text { 236. 242. 244. 257. 265. 282. 291. 308. 313. 342. 355. 374. 376. 382. 384. 391. 413. } 419 . \\
\text { 420. 424. 436. 450. 451. 471. 479. 485. 499. 508. 536. 552. 558. 568. 577. 589. 601. } 606 . \\
\text { 614. 629. 640. 647. 650. 655. 666. 668. 674. 683. 685. 702. 712. 714. 736. 741. 742. } 745 . \\
\text { 793. 795. 803. 813. 827. 837. 838. 845. 849. 850. 856. 863. 867. 870. 893. 925. 934. 954. } \\
\text { 960. 974. 988.1026. 1032. 1037. 1070. 1080. 1121. 1126. 1130. 1139. 1145. 1183. 1189. } \\
\text { 1192. 1206. 1207. 1213. 1217. 1217. 1238. 1248. 1267. 1277. 1283. 1295. 1313. 1317. } 1317 . \\
\text { 1336. 1338. 1339. 1340. 1361. 1366. 1384. 1388. 1405. 1410. 1419. 1427. 1434. 1469. } 1476 . \\
\text { 1487. 1494. 1494. 1515. 1523. 1540. 1570. 1575. 1635. 1638. 1645. 1650. 1652. 1660. } 1665 . \\
\text { 1672. 1677. 1696. 1719. 3006. 3133. 3172. 3184. 3191. 3197. 3202. 3208. 3218. 3220. } 3234 . \\
\text { 3241. 3245. 3245. 3347. 3532. 3708. 3746. 3749. 3769. }\end{array}$ \\
\hline $2 \mathrm{~b} 2$ & $\begin{array}{l}\text { 6. 16. 25. 34. 35. 42. 47. 59. 61.102. 111. 116. 121. 141. 158. 165. 182. 198. 215. 220. } 228 . \\
\text { 231. 236. 242. 255. 261. 280. 291. 305. 311. 340. 356. 368. 376. 381. 385. 395. 415. } 416 . \\
\text { 420. 423. 433. 446. 454. 462. 467. 482. 508. 511. 534. 553. 558. 566. 575. 592. 602. } 610 . \\
\text { 618. 629. 641. 644. 650. 655. 659. 665. 667. 682. 684. 703. 708. 713. 734. 740. 741. } 747 . \\
\text { 789. 794. 805. 815. 821. 831. 838. 842. 845. 852. 853. 865. 871. 873. 886. 926. 941. 949. } \\
\text { 961. 974. 991.1019. 1032. 1040. 1071. 1082. 1119. 1127. 1128. 1141. 1145. 1181. 1188. } \\
\text { 1192. 1205. 1206. 1211. 1215. 1216. 1230. 1241. 1266. 1277. 1288. 1295. 1312. 1315. 1318. } \\
\text { 1334. 1338. 1339. 1347. 1362. 1367. 1375. 1386. 1405. 1411. 1416. 1422. 1435. 1469. } 1474 . \\
\text { 1485. 1487. 1492. 1514. 1521. 1541. 1568. 1577. 1636. 1639. 1643. 1650. 1654. 1663. } 1664 . \\
\text { 1671. 1677. 1696. 1719. 3006. 3158. 3167. 3171. 3202. 3204. 3204. 3215. 3217. 3223. 3229. } \\
\text { 3242. 3245. 3250. 3346. 3542. 3709. 3746. 3750. 3769. }\end{array}$ \\
\hline $2 \mathrm{b3}$ & 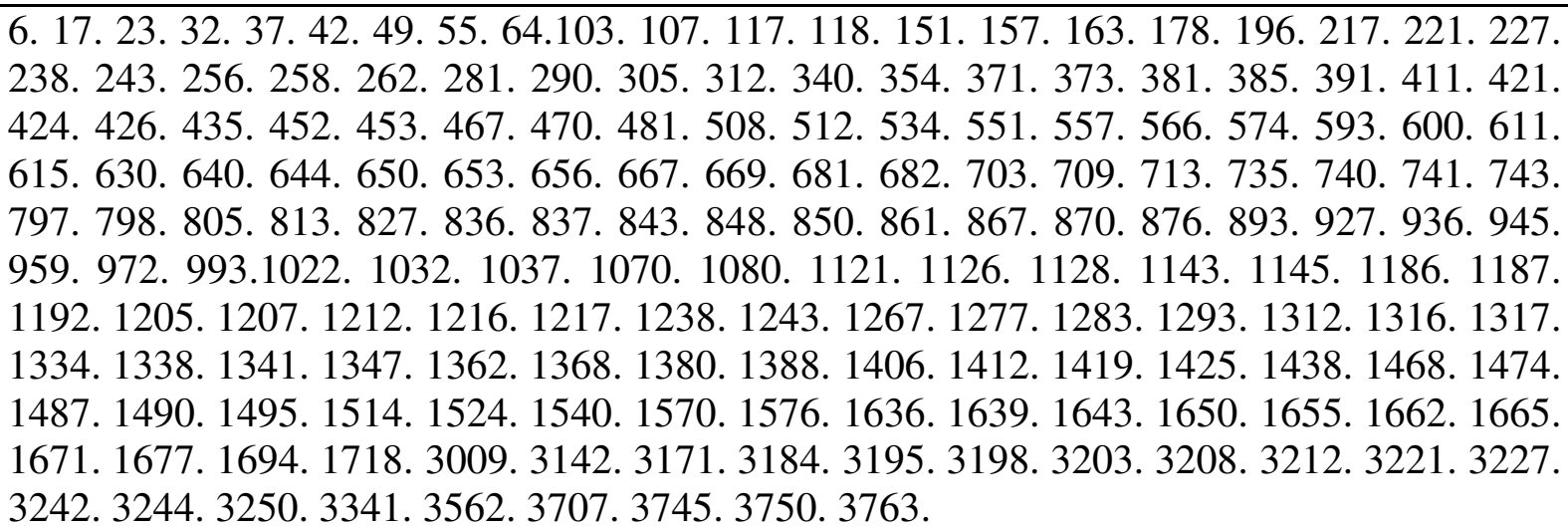 \\
\hline $2 \mathrm{~b} 4$ & 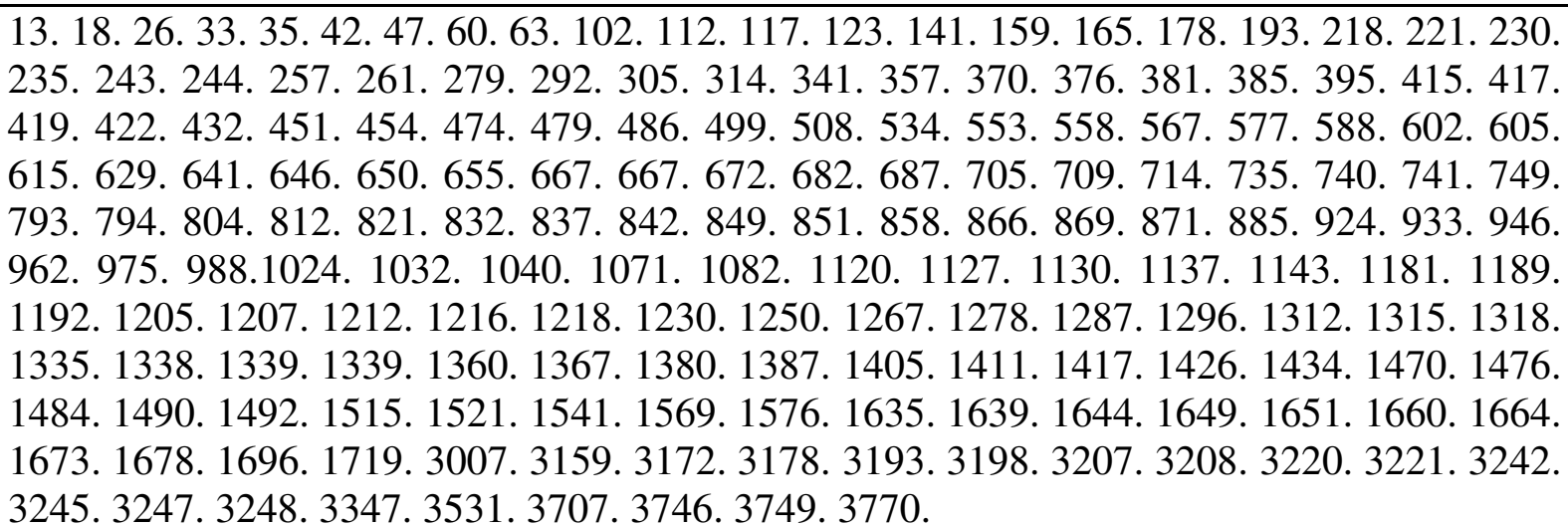 \\
\hline $2 \mathrm{b5}$ & $\begin{array}{ccccccccccccccccc}11 . & 19 . & 26 . & 29 . & 40 . & 44 . & 48 . & 54 . & 56 . & 95 . & 101 . & 109 . & 129 . & 142 . & 159 . & 168 . & 176 . \\
191 . & 218 . & 222 . & 224 . & 234 . & 237 . & 243 . & 255 . & 263 . & 285 . & 290 . & 295 . & 314 . & 339 . & 356 . & 368 . \\
\text { 371. } 374 . & 382 . & 383 . & 400 . & 413 . & 414 . & 420 . & 422 . & 436 . & 450 . & 455 . & 469 . & 480 . & 486 . & 496 . \\
507 . & 533 . & 557 . & 560 . & 568 . & 579 . & 593 . & 603 . & 611 . & 623 . & 629 . & 636 . & 647 . & 649 . & 654 . & 665 .\end{array}$ \\
\hline
\end{tabular}




\begin{tabular}{|c|c|}
\hline & $\begin{array}{l}\text { 672. 683. 685. 704. 715. 721. 733. 740. 744. 749. 790. 802. 809. 813. 822. 830. } \\
\text { 840. 843. 850. 853. 856. 866. 867. 874. 895. 935. 941. 956. 968. 980. 996. } 1031 . \\
\text { 1033. 1042. 1065. 1071. 1106. 1125. 1134. 1138. 1141. 1187. 1192. 1197. 1205. 1210. 1211. } \\
\text { 1214. 1216. 1236. 1247. 1252. 1258. 1278. 1292. 1298. 1311. 1315. 1330. 1337. 1339. 1352. } \\
\text { 1359. 1368. 1385. 1389. 1400. 1411. 1416. 1425. 1431. 1466. 1471. 1491. 1492. 1495. 1512. } \\
\text { 1549. 1557. 1568. 1573. 1635. 1644. 1650. 1652. 1662. 1662. 1672. 1677. 1685. 1704. 1720. } \\
\text { 3052. 3069. 3151. 3167. 3187. 3187. 3198. 3204. 3209. 3219. 3222. 3243. 3245. 3250. 3258. } \\
\text { 3709. 3747. 3751. 3756. 3771. }\end{array}$ \\
\hline $2 \mathrm{~b} 7$ & 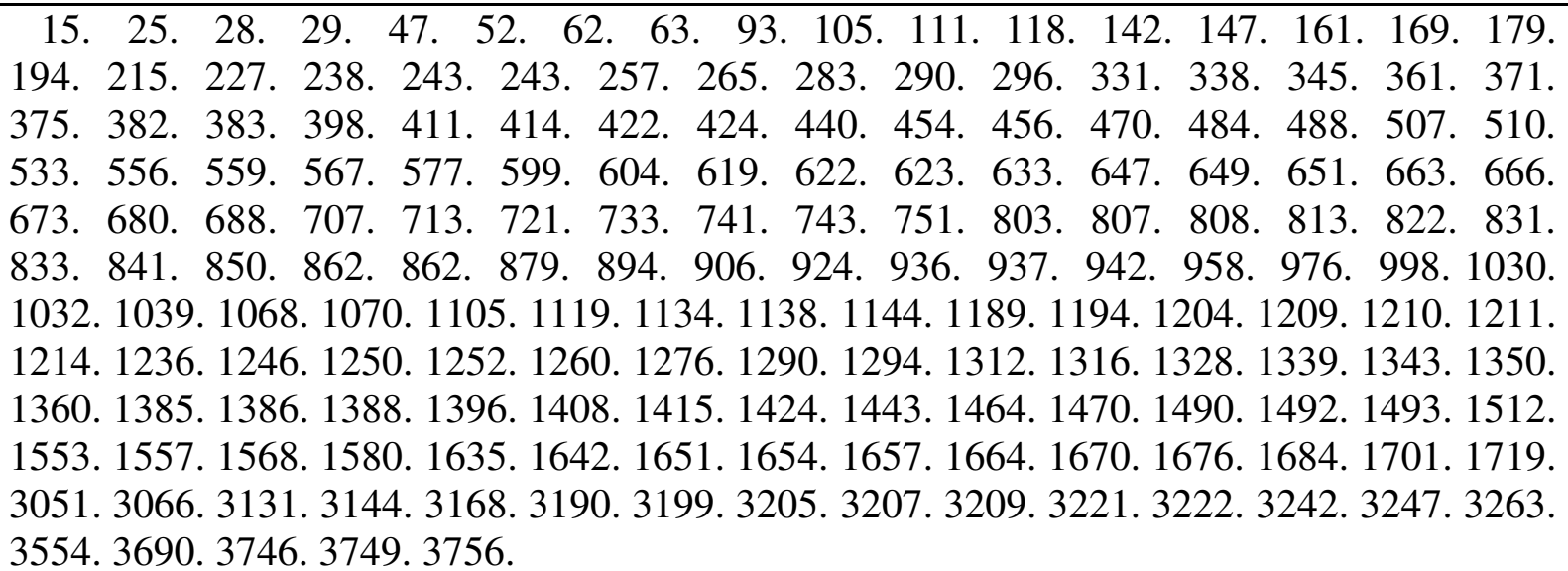 \\
\hline
\end{tabular}

TABLE S5. Dihedral Angles of Conformers of (2R,3S)-2 Optimized at B3LYP/6-31G* Level in the Gas Phase (Degree)

\begin{tabular}{c|rrrrrrrrrrrrrr}
\hline Species & $\mathbf{2 a 1}$ & $\mathbf{2 a 2}$ & $\mathbf{2 a 3}$ & $\mathbf{2 a 4}$ & $\mathbf{2 a 5}$ & $\mathbf{2 a 6}$ & $\mathbf{2 a 7}$ & $\mathbf{2 a 8}$ & $\mathbf{2 b 1}$ & $\mathbf{2 b 2}$ & $\mathbf{2 b 3}$ & $\mathbf{2 b 4}$ & $\mathbf{2 b 5}$ & $\mathbf{2 b 7}$ \\
\hline C7"-C8"-C3-C4 & 51 & 50 & 51 & 50 & 58 & 59 & 57 & 61 & -80 & -82 & -83 & -82 & -126 & -131 \\
H2-C2-C3-H3 & -170 & -170 & -171 & -171 & -178 & -176 & -179 & -174 & 180 & 174 & 178 & 174 & -175 & -177 \\
C2'-C1'-C2-O1 & 37 & 45 & 36 & 46 & 39 & 48 & 35 & 51 & 69 & 52 & 66 & 53 & 51 & 51 \\
C2”"C1'"C2"-C1" & 158 & -155 & -19 & 26 & 154 & -157 & -26 & 25 & 154 & 30 & -25 & -151 & 149 & 149 \\
O1-C9-C8-C7 & 179 & 179 & 179 & 179 & -179 & -179 & -179 & -179 & -177 & -177 & -177 & -177 & 0 & 180 \\
C4-C10-C5-C6 & 175 & 175 & 175 & 175 & 176 & 175 & 177 & 175 & 175 & 176 & 176 & 176 & 175 & 174 \\
C3-C4-C10-C5 & -170 & -170 & -171 & -171 & -175 & -174 & -175 & -174 & 0 & 179 & 178 & 179 & -175 & -179 \\
C2-O1-C9-C8 & -161 & -161 & -160 & -160 & -157 & -157 & -157 & -157 & -152 & -153 & -151 & -153 & -157 & -155 \\
\hline
\end{tabular}


TABLE S6: Important Thermodynamic Parameters of $(2 R, 3 S)-2$ in the Gas Phase and in Methanol Solution (au)

\begin{tabular}{|c|c|c|c|c|c|}
\hline \multirow[t]{2}{*}{ Species } & \multicolumn{4}{|c|}{ In Gas Phase } & \multirow{2}{*}{$\begin{array}{c}\text { In Methanol } \\
E s\end{array}$} \\
\hline & $E$ & $E^{\prime}=E+Z P E$ & $H$ & $G$ & \\
\hline $2 a 1$ & -1982.696503 & -1982.245446 & -1982.211214 & -1982.311546 & -1982.739733 \\
\hline $2 a 2$ & -1982.697149 & -1982.246044 & -1982.211842 & -1982.311927 & -1982.740402 \\
\hline $2 a 3$ & -1982.696078 & -1982.245260 & -1982.210955 & -1982.312226 & -1982.738160 \\
\hline $2 \mathrm{a} 4$ & -1982.696981 & -1982.246072 & -1982.211801 & -1982.312617 & -1982.739718 \\
\hline $2 \mathrm{a} 5$ & -1982.696311 & -1982.245698 & -1982.211178 & -1982.312381 & -1982.744035 \\
\hline $2 a 6$ & -1982.696129 & -1982.245567 & -1982.210989 & -1982.312998 & -1982.744295 \\
\hline $2 a 7$ & -1982.696459 & -1982.245832 & -1982.211301 & -1982.313625 & -1982.742245 \\
\hline $2 \mathrm{a} 8$ & -1982.696552 & -1982.245945 & -1982.211391 & -1982.313220 & -1982.743631 \\
\hline $2 \mathrm{~b} 1$ & -1982.692620 & -1982.241203 & -1982.207082 & -1982.307011 & -1982.736588 \\
\hline $2 \mathrm{~b} 2$ & -1982.688992 & -1982.237828 & -1982.203583 & -1982.304429 & -1982.730874 \\
\hline $2 \mathrm{b3}$ & -1982.693131 & -1982.241753 & -1982.207559 & -1982.308295 & -1982.734072 \\
\hline $2 \mathrm{~b} 4$ & -1982.689414 & -1982.238047 & -1982.203883 & -1982.303888 & -1982.733159 \\
\hline $2 \mathrm{b5}$ & -1982.696654 & -1982.245810 & -1982.211361 & -1982.312137 & -1982.744138 \\
\hline $2 b 7$ & -1982.706502 & -1982.254637 & -1982.220942 & -1982.318590 & -1982.747777 \\
\hline
\end{tabular}

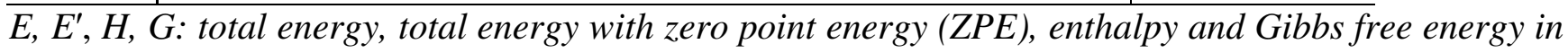
the gas phase at B3LYP/6-31G* level; Es: single point energy at B3LYP-SCRF/6-31G*//B3LYP/6$31 G^{*}$ level with COSMO model in methanol solution 


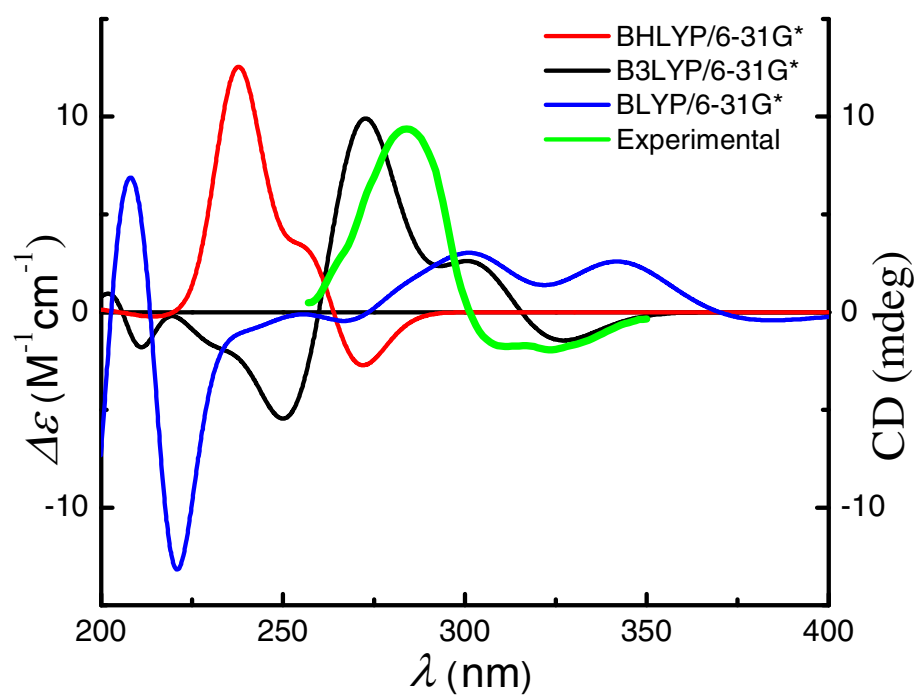

FIGURE S1. Theoretically calculated ECD spectra of $(2 R)-1$ by TDDFT at BHLYP/6-31G* and BLYP/6-31G* and its experimental ECD (in the region 260-350 nm). 

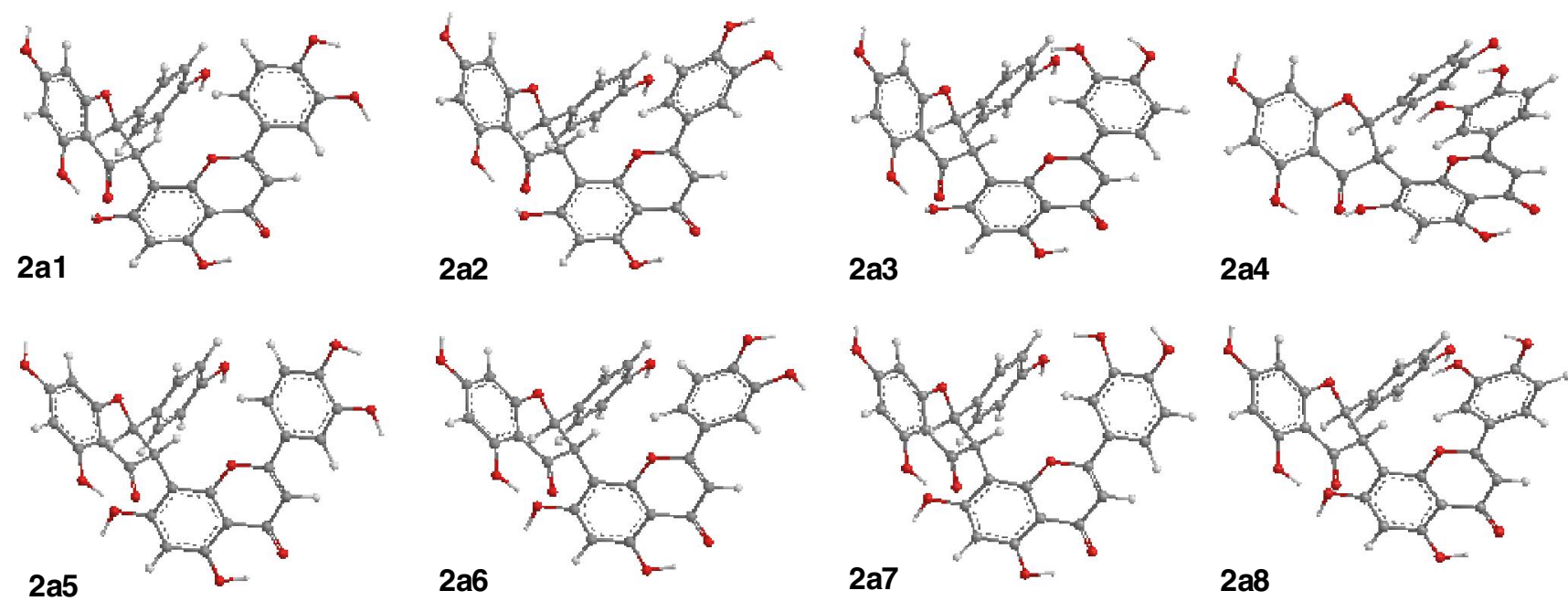

2a4

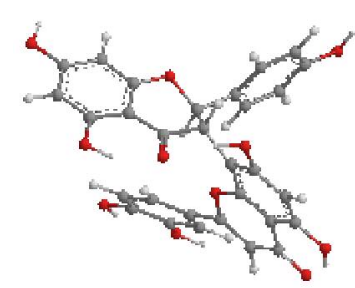

2b1

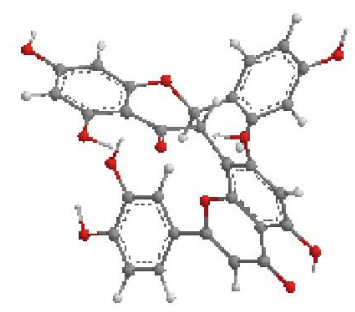

$2 b 2$

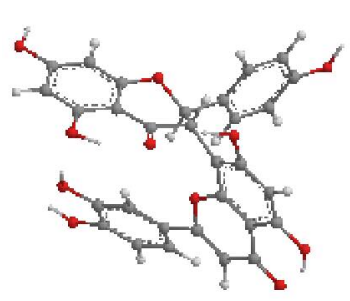

2b3

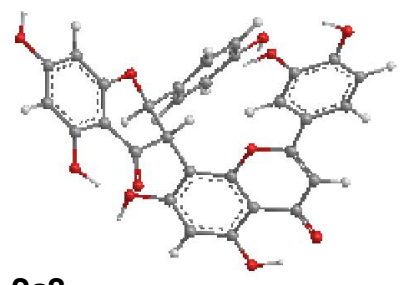

2a8
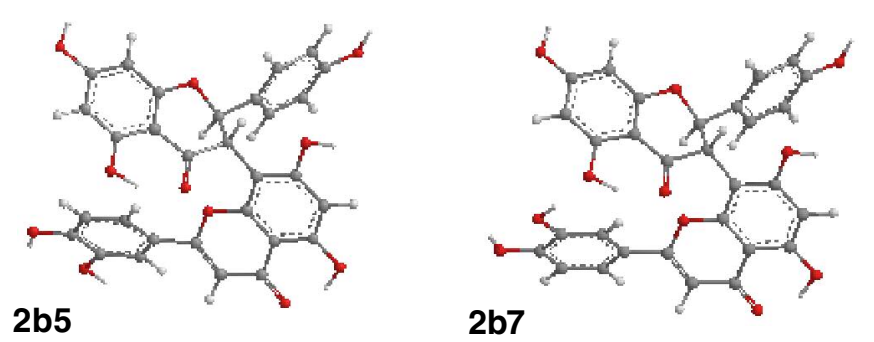

FIGURE S2. Optimized geometries of conformers of $(2 R, 3 S)-2$ at B3LYP/6-31G* level in the gas phase. 

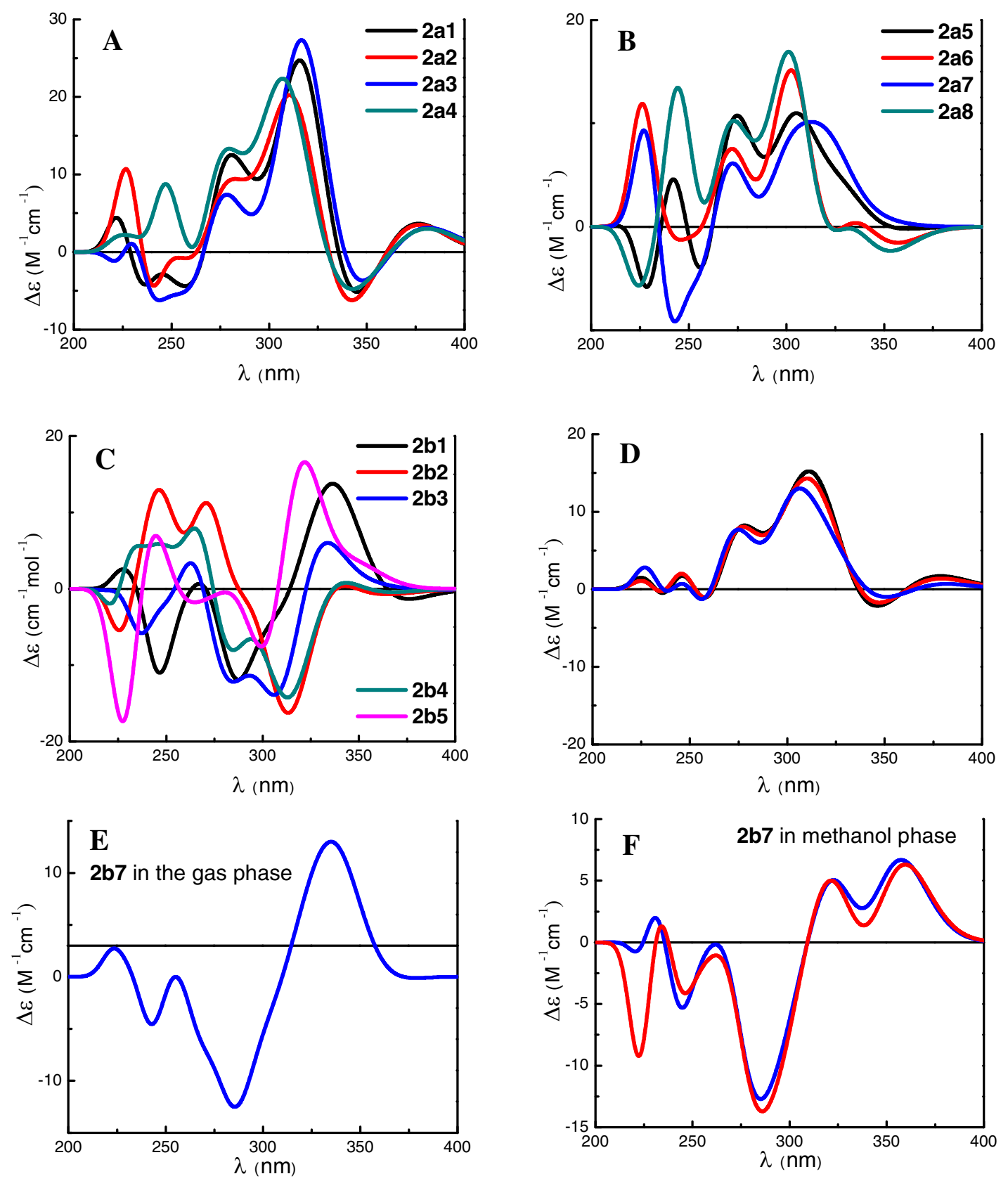

FIGURE S3. Theoretically calculated ECD spectra of $(2 R, 3 S)-\mathbf{2}(\mathbf{A} \rightarrow \mathbf{C})$ and their weighted ECD spectra (D: -, by $P_{E} \%$; by $P_{E^{\prime}} \%$; , by $P_{G} \%$ ) in the gas phase at B3LYP/6-31G* level and those of conformer $\mathbf{2 b 7}$ in the gas phase at B3LYPP/6-31G* level $(\mathbf{E})$ and in methanol with COSMO model (F: 一, at B3LYP-SCRF/6-31G*//B3LYP/6-31G* level; 一, at B3LYP-SCRF/6-31G* level). 